Pacific

Journal of

Mathematics

A GJMS CONSTRUCTION FOR 2-TENSORS AND THE SECOND VARIATION OF THE TOTAL $Q$-CURVATURE

YoshIHIKO MATSUMOTO 


\title{
A GJMS CONSTRUCTION FOR 2-TENSORS AND THE SECOND VARIATION OF THE TOTAL $Q$-CURVATURE
}

\author{
YoshIHIKO MATSUMOTO
}

\begin{abstract}
We construct a series of conformally invariant differential operators acting on weighted trace-free symmetric 2-tensors by a method similar to that of Graham, Jenne, Mason, and Sparling. For compact conformal manifolds of dimension even and greater than or equal to four with vanishing ambient obstruction tensor, one of these operators is used to describe the second variation of the total $Q$-curvature. An explicit formula for conformally Einstein manifolds is given in terms of the Lichnerowicz Laplacian of an Einstein representative metric.
\end{abstract}

\section{Introduction}

Let $(M,[g])$ be a conformal manifold of dimension $n \geq 3$. The $k$-th GJMS operator [Graham et al. 1992] is a conformally invariant differential operator acting on densities $\mathscr{E}(-n / 2+k) \rightarrow \mathscr{E}(-n / 2-k)$, which is defined for all $k \in \mathbb{Z}_{+}$if $n$ is odd and for integers within the range $1 \leq k \leq n / 2$ if $n$ is even. This operator has a universal expression in terms of any representative metric $g \in[g]$ with leading term the $k$-th power of the Laplacian. The idea for the construction is realizing densities as functions on the metric cone $\varphi$ and computing the obstruction of its formal harmonic extension to the ambient space $(\tilde{\mathscr{G}}, \tilde{g})$, where $\tilde{g}$ is an ambient metric of Fefferman and Graham [1985; 2012]. After the appearance of [Graham et al. 1992], other GJMS-like conformally invariant differential operators have been constructed in, e.g., [Branson and Gover 2005; Gover and Peterson 2006].

In this article, we establish another variant of the GJMS construction. Our operators $P_{k}$ act on weighted trace-free symmetric (covariant) 2-tensors:

$$
P_{k}: \mathscr{Y}_{0}\left(-\frac{n}{2}+2+k\right) \rightarrow \mathscr{S}_{0}\left(-\frac{n}{2}+2-k\right) .
$$

Here, the values that $k$ takes are the same as in the density case, $\mathscr{Y}_{0}$ is the space of trace-free symmetric 2-tensors on $M$, and $\mathscr{Y}_{0}(w)=\mathscr{Y}_{0} \otimes \mathscr{E}(w)$. The main tool of

Partially supported by Grant-in-Aid for JSPS Fellows (22-6494).

MSC2010: primary 53A30; secondary 53A55.

Keywords: conformal manifolds, conformally Einstein manifolds, invariant differential operators,

GJMS construction, $Q$-curvature, ambient metric, Lichnerowicz Laplacian. 
our construction is the Lichnerowicz Laplacian of the ambient metric $\tilde{g}$, which is defined by

$$
\tilde{\Delta}_{\mathrm{L}}:=\tilde{\Delta}+2 \widetilde{\operatorname{Ric}}^{\circ}-2 \stackrel{\tilde{R}}{ }
$$

where $\tilde{\Delta}=\tilde{\nabla}^{*} \tilde{\nabla}$ is the connection Laplacian and $\widetilde{\operatorname{Ric}^{\circ}}, \stackrel{\tilde{R}}{\text { are the following tensorial }}$ actions of the Ricci and Riemann curvature tensors of $\tilde{g}$ :

$$
\begin{aligned}
\left(\widetilde{\operatorname{Ric}^{\circ}} \tilde{\sigma}\right)(X, Y) & :=\frac{1}{2}\left(\langle\widetilde{\operatorname{Ric}}(X, \cdot), \tilde{\sigma}(Y, \cdot)\rangle_{\tilde{g}}+\langle\widetilde{\operatorname{Ric}}(Y, \cdot), \tilde{\sigma}(X, \cdot)\rangle_{\tilde{g}}\right), \\
(\tilde{R} \tilde{\sigma})(X, Y) & :=\langle\tilde{R}(X, \cdot, Y, \cdot), \tilde{\sigma}\rangle_{\tilde{g}} .
\end{aligned}
$$

Our intention to study the GJMS construction for 2-tensors is because of its relation to the second variation of the total $Q$-curvature, i.e., the integral of Branson's $Q$-curvature [1995]. Recall that, for a 4-dimensional compact conformal manifold $(M,[g])$ of positive definite signature, the Chern-Gauss-Bonnet formula for the total $Q$-curvature $\bar{Q}$ is

$$
\bar{Q}=8 \pi^{2} \chi(M)-\frac{1}{4} \int_{M}|W|_{g}^{2} d V_{g},
$$

where $\chi(M)$ is the Euler characteristic, $W$ is the Weyl tensor, and $g \in[g]$ is any representative. One can deduce from this that $\bar{Q} \leq 8 \pi^{2} \chi(M)$ and the equality holds if and only if $(M,[g])$ is conformally flat. It turns out that there is a partial generalization of this fact to the higher dimensions. Recall that a conformal metric $[g]$ is identified with a weighted 2-tensor $g \in \mathscr{S}(2)$. Let $\mathscr{K}_{[g]}$ be the conformal Killing operator. Then we have the following theorem, which is due to Møller and Ørsted [2009].

Theorem 0.1. Let $S^{n}$ be the sphere of even dimension $n \geq 4$. Then, for any smooth 1-parameter family $\boldsymbol{g}_{t}$ of conformal metrics on $S^{n}$ such that $\boldsymbol{g}_{0}=\boldsymbol{g}_{\text {std }}$ and $\left.\dot{\boldsymbol{g}}_{t}\right|_{t=0} \notin$ image $\mathcal{K}_{\left[g_{\text {std }}\right]}$, the total $Q$-curvature $\bar{Q}_{t}$ attains a local maximum at $t=0$.

Our main theorem contains Theorem 0.1 as a special case. Consider the following decomposition of $\mathscr{Y}_{0}(2)$, which is valid for any compact positive definite conformal manifold $(M,[g])$ and a representative $g \in[g]$ (see [Besse 1987, Section 12.21]):

$$
\mathscr{Y}_{0}(2)=\text { image } \mathcal{K}_{[g]} \oplus \mathscr{Y}_{\mathrm{TT}}^{g}(2) .
$$

Here $\mathscr{Y}_{\mathrm{TT}}^{g}(w)$ is the space of TT-tensors (trace-free and divergence-free tensors) with respect to $g$. This is an orthogonal decomposition with respect to the $L^{2}$-inner product, and if $g$ is Einstein, the Lichnerowicz Laplacian $\Delta_{\mathrm{L}}$ of $g$ respects this decomposition.

Theorem 0.2. Let $(M,[g])$ be a compact conformally Einstein manifold of positive definite signature with even dimension $n \geq 4$, and $g$ an Einstein representative with Schouten tensor $P_{i j}=\lambda g_{i j}$. Then, for any smooth 1-parameter family $\boldsymbol{g}_{t}$ of 
conformal metrics such that $\boldsymbol{g}_{0}=\mathbf{g}$, the second derivative of the total $Q$-curvature at $t=0$ is

$$
\left.\frac{d^{2}}{d t^{2}} \bar{Q}_{t}=-\frac{1}{4} \int_{M} \int_{m=0}^{n / 2-1}\left(\Delta_{\mathrm{L}}-4(n-1) \lambda+4 m(n-2 m-1) \lambda\right) \varphi_{\mathrm{TT}}^{g}, \varphi_{\mathrm{TT}}^{g}\right\rangle_{g}
$$

where $\varphi_{\mathrm{TT}}^{g}$ is the $\varphi_{\mathrm{TT}}^{g}(2)$-component of $\varphi=\left.\dot{\boldsymbol{g}}_{t}\right|_{t=0}$ with respect to (0-1). In particular, suppose there is an Einstein representative $g$ with $\lambda \geq 0$ such that the smallest eigenvalue $\alpha$ of $\left.\Delta_{\mathrm{L}}\right|_{\mathscr{T}_{\mathrm{T}}^{g}(2)}$ satisfies

$$
\alpha>4(n-1) \lambda .
$$

Then, for any $\boldsymbol{g}_{t}$ for which $\varphi \notin$ image $\mathscr{K}_{[g]}$, the total $Q$-curvature attains a local maximum at $t=0$.

For $\left(S^{n}, g_{\text {std }}\right), \lambda=1 / 2$ and $\Delta_{\mathrm{L}}=\Delta+2 n$. Therefore the assumption for the latter half of Theorem 0.2 is satisfied, and hence Theorem 0.1 follows.

Some ideas for the proof of Theorem 0.2 are in order. Let $(M,[g])$ be a compact conformal manifold of even dimension $n \geq 4$ (here we may allow arbitrary signature). If we are given a smooth family $\boldsymbol{g}_{t}$ of conformal metrics on $M$ such that $\boldsymbol{g}_{0}=\boldsymbol{g}$, then the derivative $\varphi_{t}=\dot{\boldsymbol{g}}_{t} \in \mathscr{Y}(2)$ is trace-free with respect to $\boldsymbol{g}_{t}$. As shown in [Graham and Hirachi 2005], the derivative of $\bar{Q}_{t}$ is given by

$$
\frac{d}{d t} \bar{Q}_{t}=(-1)^{n / 2} \frac{n-2}{2} \int_{M}\left\langle\mathcal{O}_{t}, \varphi_{t}\right\rangle_{g_{t}},
$$

where $\mathscr{O}_{t}$ is the Fefferman-Graham ambient obstruction tensor of $\boldsymbol{g}_{t}$ [Fefferman and Graham 1985; 2012]. In particular, if $(M,[g])$ has vanishing obstruction tensor, which is the case if $(M,[g])$ is conformally Einstein for instance, then $\bar{Q}_{t}$ stabilizes at $t=0$. In this case the second derivative of $\bar{Q}_{t}$ at $t=0$ is of interest. It is given by

$$
\left.\frac{d^{2}}{d t^{2}} \bar{Q}_{t}\right|_{t=0}=(-1)^{n / 2} \frac{n-2}{2} \int_{M}\left\langle\mathcal{O}_{g}^{\prime} \varphi, \varphi\right\rangle_{g},
$$

where $\mathscr{O}_{g}^{\prime}: \mathscr{Y}_{0}(2) \rightarrow \mathscr{Y}_{0}(2-n)$ is the linearization at $\boldsymbol{g}$ of the obstruction tensor operator $\left(\widetilde{O}_{g}^{\prime} \varphi\right.$ is trace-free because $g$ is obstruction-flat). This shows that it suffices to compute $\mathrm{O}_{g}^{\prime}$ to derive the second variational formula of the total $Q$-curvature. The construction of our operators $P_{k}$ leads to the fact that $P=P_{n / 2}$ is equal to $O_{g}^{\prime}$ up to a constant factor for obstruction-flat manifolds. (For $n=4$ and 6 , since an explicit formula of the obstruction tensor is known, one can directly compute its linearization. In higher dimensions our result is really new, because there is no such concrete formula for $\mathrm{O}^{\text {.) }}$ Thus our GJMS construction adds new knowledge of $\mathbb{O}_{g}^{\prime}$, which was previously studied in [Branson 2005; Branson and Gover 2007; 2008]. 
If we specialize to the case of conformally Einstein manifolds, explicit computation is possible thanks to a well-known associated ambient metric. We will derive a formula of $P_{k}$ restricted to $\mathcal{S}_{\mathrm{TT}}^{g}(-n / 2+2+k)$ with respect to an Einstein representative $g$ with Schouten tensor $P_{i j}=\lambda g_{i j}$ :

(0-5) $\left.P_{k}\right|_{\varphi_{\mathrm{TT}}^{g}(-n / 2+2+k)}=\prod_{m=0}^{k-1}\left(\Delta_{\mathrm{L}}-4(n-1) \lambda-2\left(-\frac{n}{2}+k-2 m\right)\left(\frac{n}{2}+k-2 m-1\right) \lambda\right)$.

Then Theorem 0.2 is an immediate consequence.

This article is organized as follows. Preliminaries about ambient metrics and some preparatory lemmas are included in Section 1. In Section 2, our operators $P_{k}$ are constructed. One of the characterizations of $P_{k}$ is that it gives the obstruction to dilation-annihilating TT-harmonic extension of $\varphi \in \mathscr{Y}_{0}(-n / 2+2+k)$ with respect to the ambient Lichnerowicz Laplacian $\tilde{\Delta}_{\mathrm{L}}$. In Section 3, we first show that the variation of the normal-form ambient metric modified by adding a certain tensor in the image of the Killing operator of $\tilde{g}$ is a best possible approximate solution to the harmonic extension problem mentioned above. Using this fact, we prove that the trace-free part of $O_{g}^{\prime}$ equals $P$ in general. In Section 4, we work on conformally Einstein manifolds and prove Theorem 0.2.

In this article, "conformal manifolds" are of arbitrary signature unless otherwise stated. Index notation is used throughout. On ambient spaces we use $I, J, K, \ldots$ as indices, while on the original manifolds $i, j, k, \ldots$ are used.

\section{Preliminaries}

Let $(M,[g])$ be a conformal manifold of dimension $n$ of signature $(p, q)$ with metric cone $\mathscr{G}$. With a fixed representative metric $g \in[g], \mathscr{G}$ is trivialized as

$$
\mathscr{G} \cong \mathbb{R}_{+} \times M, \quad t^{2} g_{x} \mapsto(t, x) .
$$

Let $\tilde{\mathscr{G}}$ be the associated ambient space:

$$
\tilde{\varphi}:=\varphi \times \mathbb{R} \cong \mathbb{R}_{+} \times M \times \mathbb{R}=\{(t, x, \rho)\} .
$$

In our index notation, if $\tilde{\mathscr{G}}$ is trivialized as above, we use the indices 0 and $\infty$ for the $t$ - and $\rho$-components, respectively.

The space $\mathcal{G}_{\mathcal{C}}$ carries a natural $\mathbb{R}_{+}$-bundle structure. The dilation $\delta_{s}, s \in \mathbb{R}^{\times}$, is by definition the action of $s^{2} \in \mathbb{R}_{+}$, and the infinitesimal dilation field is denoted by $T$. The spaces of the densities, weighted 1-forms, and weighted covariant symmetric 2-tensors (all of weight $w$ ) are denoted by $\mathscr{E}(w), \mathscr{T}(w)$, and $\mathscr{S}(w)$. By the metric 
cone $\mathscr{G}$, these spaces are realized as follows:

$$
\begin{aligned}
& \mathscr{E}(w)=\left\{f \in C^{\infty}(\mathscr{G}, \mathbb{R}) \mid T f=w f\right\}, \\
& \left.\mathscr{T}(w)=\left\{\tau \in C^{\infty}\left(\mathscr{G}, T^{*} \mathscr{G}\right) \mid T\right\lrcorner \tau=0, \quad \mathscr{L}_{T} \tau=w \tau\right\}, \\
& \left.\mathscr{S}(w)=\left\{\sigma \in C^{\infty}\left(\mathscr{G}, \operatorname{Sym}^{2} T^{*} \mathscr{G}\right) \mid T\right\lrcorner \sigma=0, \quad \mathscr{L}_{T} \sigma=w \sigma\right\} .
\end{aligned}
$$

The $\mathbb{R}_{+}$-action extends to $\tilde{\mathscr{G}}=\mathscr{G} \times \mathbb{R}$ and so does $T$. In terms of the extended $T$, we define

$$
\begin{aligned}
& \tilde{\mathscr{E}}(w):=\left\{\tilde{f} \in C^{\infty}(\tilde{\mathscr{G}}, \mathbb{R}) \mid T \tilde{f}=w \tilde{f}\right\}, \\
& \tilde{\mathscr{T}}(w):=\left\{\tilde{\tau} \in C^{\infty}\left(\tilde{\mathscr{G}}, T^{*} \tilde{\mathscr{G}}\right) \mid \mathscr{L}_{T} \tilde{\tau}=w \tilde{\tau}\right\}, \\
& \tilde{\mathscr{S}}(w):=\left\{\tilde{\sigma} \in C^{\infty}\left(\tilde{\mathscr{G}}, \operatorname{Sym}^{2} T^{*} \tilde{\mathscr{G}}\right) \mid \mathscr{L}_{T} \tilde{\sigma}=w \tilde{\sigma}\right\} .
\end{aligned}
$$

When $\tilde{\sigma} \in \tilde{\mathscr{T}}(w)$ satisfies $(T\lrcorner \tilde{\sigma})\left.\right|_{T^{\varphi}}=0$, then $\left.\tilde{\sigma}\right|_{T^{\varphi}}$ makes sense as a section in $\mathscr{Y}(w)$ via the identification (1-1). We use the notation $\left.\tilde{\sigma}\right|_{T M}$ to express this weighted tensor.

Let $\tilde{g}$ be a preambient metric. This means that $\tilde{g} \in \tilde{\mathscr{T}}(2)$ is a homogeneous pseudoRiemannian metric of signature $(p+1, q+1)$ defined on a dilation-invariant open neighborhood of $\mathscr{G}$ in $\tilde{G}$ such that its pullback to $\mathscr{G}$ is equal to $g \in \mathscr{Y}(2)$. In the sequel we only work asymptotically near $\mathscr{G}$, so we may assume that all preambient metrics are defined on the whole $\tilde{\mathscr{G}}$. We next introduce the straightness condition:

$$
\tilde{\nabla} T=\mathrm{id} .
$$

If this is true, the differential of the canonical defining function $r=|T|_{\tilde{g}}^{2}$ of $\mathscr{G}_{\text {is }}$

$$
d r=2 T\lrcorner \tilde{g} .
$$

Recall that it follows immediately from (1-2) that

$$
T^{I} \tilde{R}_{I J K L}=0, \quad \text { and hence } \quad T^{I} \widetilde{\operatorname{Ric}}_{I J}=0 .
$$

The Fefferman-Graham theorem states that there is a straight preambient metric $\tilde{g}$ with

$$
\widetilde{\mathrm{Ric}}= \begin{cases}O\left(r^{\infty}\right) & \text { if } n \text { is odd, } \\ O\left(r^{n / 2-1}\right) & \text { if } n \text { is even. }\end{cases}
$$

In this article, such a metric $\tilde{g}$ is called an ambient metric. When $n$ is odd, ambient metrics are unique modulo $O\left(r^{\infty}\right)$ and the action of dilation-invariant diffeomorphisms on $\tilde{\mathscr{G}}$ leaving points on $\mathscr{G}$ fixed (such diffeomorphisms are called ambient-equivalence maps in the sequel). If $n$ is even, the situation is subtle. For a 1-form $\tilde{\tau} \in \tilde{\mathscr{T}}(w)$, we define

$$
\begin{aligned}
\tilde{\tau}=O^{-}\left(r^{m}\right) & \Longleftrightarrow \tilde{\tau}=O\left(r^{m-1}\right) \quad \text { and }\left.\quad\left(r^{1-m} \tilde{\tau}\right)\right|_{T^{\varphi} \text { vanishes }} \\
& \left.\Longleftrightarrow \tilde{\tau}=O\left(r^{m}\right) \quad \bmod r^{m-1} T\right\lrcorner \tilde{g} .
\end{aligned}
$$


We say that $\tilde{\sigma} \in \tilde{\mathscr{T}}(w)$ is $O^{+}\left(r^{m}\right)$ if

(i) $\tilde{\sigma}=O\left(r^{m}\right)$;

(ii) $T\lrcorner \tilde{\sigma}=O^{-}\left(r^{m+1}\right)$ and hence $\left.\left(r^{-m} \tilde{\sigma}\right)\right|_{T M}$ makes sense; and

(iii) $\left.\left(r^{-m} \tilde{\sigma}\right)\right|_{T M} \in \mathscr{Y}(w-2 m)$ is trace-free with respect to $g$.

Then, ambient metrics are unique modulo $O^{+}\left(r^{n / 2}\right)$ and the action of ambientequivalence maps. By [Fefferman and Graham 2012, Equation (3.13)], the condition $\widetilde{\mathrm{Ric}}=O\left(r^{n / 2-1}\right)$ for ambient metrics actually forces

$$
\widetilde{\operatorname{Ric}}=O^{+}\left(r^{n / 2-1}\right) \text {. }
$$

Let $g \in[g]$ and consider the induced trivialization $\tilde{\mathscr{G}} \cong \mathbb{R}_{+} \times M \times \mathbb{R}$. If a straight preambient metric $\tilde{g}$ is near $\mathscr{G}$ of the form

$$
\tilde{g}=2 \rho d t^{2}+2 \rho d t d \rho+t^{2} g_{\rho},
$$

where $g_{\rho}$ is a 1-parameter family of metrics on $M$ with $g_{0}=g$, then $\tilde{g}$ is said to be in normal form relative to $g$. For any straight preambient metric $\tilde{g}$ and a choice of $g \in[g]$, it is known [ibid., Proposition 2.8] that there exists an ambient-equivalence map $\Phi$ such that $\Phi^{*} \tilde{g}$ is in normal form relative to $g$.

Lemma 1.1. Let $\tilde{g}$ be a straight preambient metric. For $\tilde{\tau} \in \tilde{\mathscr{T}}(w)$ and $\tilde{\sigma} \in \tilde{\mathscr{T}}(w)$,

$$
\tilde{\nabla}_{T} \tilde{\tau}=(w-1) \tilde{\tau}, \quad \tilde{\nabla}_{T} \tilde{\sigma}=(w-2) \tilde{\sigma} .
$$

Proof. Let $\tilde{\xi} \in \mathfrak{X}(\tilde{\varphi})$. Then, since the Levi-Civita connection is torsion-free,

$$
\begin{aligned}
\left(\tilde{\nabla}_{T} \tilde{\tau}\right)(\tilde{\xi}) & =T(\tilde{\tau}(\tilde{\xi}))-\tilde{\tau}\left(\tilde{\nabla}_{T} \tilde{\xi}\right)=T(\tilde{\tau}(\tilde{\xi}))-\tilde{\tau}\left([T, \tilde{\xi}]+\tilde{\nabla}_{\tilde{\xi}} T\right) \\
& =T(\tilde{\tau}(\tilde{\xi}))-\tilde{\tau}\left(\mathscr{L}_{T} \tilde{\xi}\right)-\tilde{\tau}\left(\tilde{\nabla}_{\tilde{\xi}} T\right)=\left(\mathscr{L}_{T} \tilde{\tau}\right)(\tilde{\xi})-\tilde{\tau}(\tilde{\xi})=(w-1) \tilde{\tau}(\tilde{\xi}) .
\end{aligned}
$$

The second equality is proved similarly.

Now let $\tilde{g}$ be a fixed ambient metric. Let $\tilde{\mathscr{Y}}_{0}(w)$ be the subspace of formally tracefree tensors of $\tilde{\mathscr{S}}(w)$, and $\tilde{\mathscr{S}}_{\mathrm{TT}}(w)$ the subspace of formally TT-tensors. Moreover, we define

$$
\begin{gathered}
\left.\tilde{\mathscr{S}}^{X}(w):=\{\tilde{\sigma} \in \tilde{\mathscr{T}}(w) \mid T\lrcorner \tilde{\sigma}=O\left(r^{\infty}\right)\right\}, \\
\tilde{\mathscr{Y}}_{0}^{X}(w):=\tilde{\mathscr{Y}}_{0}(w) \cap \tilde{\mathscr{S}}^{X}(w), \quad \tilde{\mathscr{S}}_{\mathrm{TT}}^{X}(w):=\tilde{\mathscr{Y}}_{\mathrm{TT}}(w) \cap \tilde{\mathscr{S}}^{X}(w) .
\end{gathered}
$$

If $n$ is odd, these spaces are invariant under $O\left(r^{\infty}\right)$-modifications of $\tilde{g}$. If $n$ is even, we need some technically defined tensor spaces. For $2-n \leq w \leq 2$, we set

$$
\tilde{\mathscr{Y}}_{\mathrm{aTT}}(w):=\left\{\tilde{\sigma} \in \tilde{\mathscr{S}}(w) \mid \operatorname{tr}_{\tilde{g}} \tilde{\sigma}=O\left(r^{\lceil(n-2+w) / 2\rceil}\right), \quad \delta_{\tilde{g}} \tilde{\sigma}=O^{-}\left(r^{\lceil(n-2+w) / 2\rceil}\right)\right\}
$$

("aTT" is for "approximately TT") and

$$
\left.\tilde{\mathscr{Y}}_{\mathrm{aTT}}^{X}(w):=\left\{\tilde{\sigma} \in \tilde{\mathscr{Y}}_{\mathrm{aTT}}(w) \mid T\right\lrcorner \tilde{\sigma}=O^{-}\left(r^{\lceil(n-2+w) / 2\rceil+1}\right)\right\},
$$


where $\delta_{\tilde{g}}$ is the divergence operator $\left(\delta_{\tilde{g}} \tilde{\sigma}\right)_{I}=-\tilde{\nabla}^{J} \tilde{\sigma}_{I J}$, and $\lceil x\rceil$ is the smallest integer not less than $x$. Then $\tilde{\mathscr{S}}_{\text {aTT }}^{X}(w)$ does not depend on the $O^{+}\left(r^{n / 2}\right)$-ambiguity of $\tilde{g}$. To check this, let $\tilde{g}^{\prime}=\tilde{g}+A$ be another ambient metric with $A=O^{+}\left(r^{n / 2}\right)$. Then $T\lrcorner A=O^{-}\left(r^{n / 2+1}\right)$. Since $\operatorname{tr}_{\tilde{g}^{\prime}} \tilde{\sigma}=\operatorname{tr}_{\tilde{g}} \tilde{\sigma}+O\left(r^{n / 2}\right)$ for any $\tilde{\sigma}$, the trace condition is not affected. The Christoffel symbol of $\tilde{g}^{\prime}$ is given by

$$
\left(\tilde{\Gamma}^{\prime}\right)_{I J}^{K}=\tilde{\Gamma}_{I J}^{K}-\frac{1}{2}\left(\tilde{g}^{\prime-1}\right)^{K L}(D A)_{L I J}=\tilde{\Gamma}_{I J}^{K}-\frac{1}{2}(D A)^{K}{ }_{I J}+O\left(r^{n / 2}\right),
$$

where

$$
(D A)_{K I J}=\tilde{\nabla}_{K} A_{I J}-\tilde{\nabla}_{I} A_{K J}-\tilde{\nabla}_{J} A_{K I} .
$$

Hence

$$
\left(\delta_{\tilde{g}^{\prime}} \tilde{\sigma}\right)_{I}=\left(\delta_{\tilde{g}} \tilde{\sigma}\right)_{I}+\frac{1}{2}(D A)^{J K}{ }_{I} \tilde{\sigma}_{J K}+\frac{1}{2}(D A)^{J K}{ }_{K} \tilde{\sigma}_{I J}+O\left(r^{n / 2}\right) .
$$

Let $A=r^{n / 2} \bar{A}$. Then

$$
(D A)_{K I J}=n r^{n / 2-1}\left(T_{K} \bar{A}_{I J}-T_{I} \bar{A}_{K J}-T_{J} \bar{A}_{K I}\right)+O\left(r^{n / 2}\right)
$$

and, because $T\lrcorner \bar{A}=O^{-}(r)$,

$$
(D A)_{K I}^{I}=n r^{n / 2-1} T_{K} \bar{A}_{I}^{I}+O^{-}\left(r^{n / 2}\right) .
$$

Therefore, if $\tilde{\sigma} \in \tilde{\mathscr{Y}}_{\mathrm{aTT}}^{X}(w), \delta_{\tilde{g}^{\prime}} \tilde{\sigma}=\delta_{\tilde{g}} \tilde{\sigma}+O^{-}\left(r^{n / 2}\right)=O^{-}\left(r^{\lceil(n-2+w) / 2\rceil}\right)$.

Lemma 1.2. Let $\tilde{g}$ be an ambient metric and $\varphi \in \mathscr{S}_{0}(-n / 2+2+k)$, where $k \in \mathbb{Z}_{+}$. If $n$ is odd, then there exists $\tilde{\sigma} \in \tilde{\mathscr{Y}}_{\mathrm{TT}}^{X}(-n / 2+2+k)$ such that $\left.\tilde{\sigma}\right|_{T M}=\varphi$. If $n$ is even, there exists $\tilde{\sigma} \in \tilde{\mathscr{T}}_{\mathrm{aTT}}^{X}(-n / 2+2+k)$ such that $\left.\tilde{\sigma}\right|_{T M}=\varphi$ as long as $k \leq n / 2$. In both cases, the restriction $\tilde{\varphi}=\left.\tilde{\sigma}\right|_{\varphi}$ is uniquely determined.

Proof. To prove the existence part, take any $\tilde{\sigma}_{(0)} \in \tilde{\mathscr{Y}}_{0}^{X}(-n / 2+2+k)$ for which $\left.\tilde{\sigma}_{(0)}\right|_{T M}=\varphi$. We shall inductively construct $\tilde{\sigma}_{(m)} \in \tilde{\mathscr{T}}_{0}^{X}(-n / 2+2+k)$ for nonnegative integers $m$ such that

$$
\tilde{\sigma}_{(m)}=\tilde{\sigma}_{(m-1)}+O\left(r^{m-1}\right), \quad \delta_{\tilde{g}} \tilde{\sigma}_{(m)}=O\left(r^{m}\right) .
$$

Suppose we have $\tilde{\sigma}_{(m-1)} \in \tilde{\mathscr{Y}}_{0}^{X}(-n / 2+2+k)$ with $\delta_{\tilde{g}} \tilde{\sigma}_{(m-1)}=O\left(r^{m-1}\right)$. If $\tilde{\sigma}_{(m)} \in$ $\tilde{\mathscr{Y}}_{0}^{X}(-n / 2+2+k)$, then $\left.T\right\lrcorner \delta_{\tilde{g}} \tilde{\sigma}_{(m)}=0$ is automatically guaranteed:

$T^{I} \tilde{\nabla}^{J}\left(\tilde{\sigma}_{(m-1)}\right)_{I J}=\tilde{\nabla}^{J}\left(T^{I}\left(\tilde{\sigma}_{(m-1)}\right)_{I J}\right)-\left(\tilde{\nabla}^{J} T^{I}\right)\left(\tilde{\sigma}_{(m-1)}\right)_{I J}=0+\tilde{g}^{I J}\left(\tilde{\sigma}_{(m-1)}\right)_{I J}=0$.

We seek for $\tilde{\sigma}_{(m)}$ assuming that it is of the form

$$
\left(\tilde{\sigma}_{(m)}\right)_{I J}=\left(\tilde{\sigma}_{(m-1)}\right)_{I J}+2 r^{m-1} T_{(I} V_{J)}+r^{m-1} \tilde{f} T_{I} T_{J}-r^{m} W_{I J},
$$

where $V \in \tilde{\mathscr{T}}(-n / 2+2+k-2 m)$ satisfies $T^{I} V_{I}=0, \tilde{f} \in \tilde{\mathscr{E}}(-n / 2+k-2 m)$, and $W \in \tilde{\mathscr{S}}^{X}(-n / 2+2+k-2 m)$ is such that the whole expression (1-6) is trace-free 
and vanishes if contracted with $T$ (hence $\operatorname{tr}_{\tilde{g}} W=\tilde{f}, T^{J} W_{I J}=V_{I}+\tilde{f} T_{I}$ ). Minus of the divergences of the additional three terms on the right-hand side of (1-6) are

$$
\begin{aligned}
\tilde{\nabla}^{J}\left(2 r^{m-1} T_{(I} V_{J)}\right) & =r^{m-1} \cdot\left((n / 2+2+k) V_{I}+T_{I} \tilde{\nabla}^{J} V_{J}\right)+O\left(r^{m}\right), \\
\tilde{\nabla}^{J}\left(r^{m-1} \tilde{f} T_{I} T_{J}\right) & =r^{m-1} \cdot(n / 2+1+k) \tilde{f} T_{I}+O\left(r^{m}\right), \\
\tilde{\nabla}^{J}\left(-r^{m} W_{I J}\right) & =r^{m-1} \cdot(-2 m)\left(V_{I}+\tilde{f} T_{I}\right)+O\left(r^{m}\right) .
\end{aligned}
$$

Therefore, we first put $V=(n / 2+2+k-2 m)^{-1} r^{-m+1} \delta_{\tilde{g}} \tilde{\sigma}_{(m-1)}$, and set $\tilde{f}=$ $-(n / 2+1+k-2 m)^{-1} \tilde{\nabla}^{J} V_{J}$ so that the $O\left(r^{m-1}\right)$-term of the divergence of (1-6) vanishes. This is possible for all $m$ if $n$ is odd, and until $m=\lfloor n / 2+k\rfloor$ if $n$ is even. Applying Borel's lemma, the proof of the existence for $n$ odd is complete. When $n$ is even, we get $\tilde{\sigma}=\tilde{\sigma}_{(\lfloor(n / 2+k) / 2\rfloor)}$. Furthermore, if $n / 2+1+k$ is an even number, then $\delta_{\tilde{g}} \tilde{\sigma}$ can be made $O^{-}\left(r^{(n / 2+1+k) / 2}\right)$. Anyway, $\delta_{\tilde{g}} \tilde{\sigma}$ finally becomes $O^{-}\left(r^{\lceil(n / 2+k) / 2\rceil}\right)$, and the existence for $n$ even is proved.

Let us once again take $\tilde{\sigma}_{(0)}$ as we did in the beginning of this proof. If $\tilde{\sigma}$ is as in the statement, then since $(T\lrcorner \tilde{\sigma})\left.\right|_{\varphi}=0$ and $\left.\tilde{\sigma}\right|_{T M}=\varphi, \tilde{\sigma}$ must be written as

$$
\tilde{\sigma}=\tilde{\sigma}_{(0)}+2 T_{(I} V_{J)}-r W_{I J},
$$

where $T^{I} V_{I}=O(r)$. Moreover, in order for $\left.T\right\lrcorner \tilde{\sigma}=O\left(r^{2}\right)$ to be satisfied, $T^{J} W_{I J}$ should be $V_{I}+r^{-1} T_{I} T^{J} V_{J}+O(r)$. Then

$$
\tilde{\nabla}^{J}\left(2 T_{(I} V_{J)}-r W_{I J}\right)=\left(\frac{n}{2}+k\right) V_{I}+T_{I}\left(\tilde{\nabla}^{J} V_{J}-2 r^{-1} T^{J} V_{J}\right)+O(r) .
$$

Therefore, $V_{I} \bmod O^{-}(r)$ is determined by the condition $\delta_{\tilde{g}} \tilde{\sigma}=O^{-}(r)$. If we put $\tilde{f} T_{I}$ into $V_{I}$, then the right-hand side will be $(n+2 k-2) \tilde{f} T_{I}$. Thus $V_{I}$ is uniquely determined in order to satisfy $\delta_{\tilde{g}} \tilde{\sigma}=O(r)$.

We call $\tilde{\varphi}$ in Lemma 1.2 the ambient lift of $\varphi \in \mathscr{Y}(-n / 2+2+k)$.

\section{A GJMS construction for trace-free symmetric 2-tensors}

Let $(M,[g])$ be a conformal manifold of dimension $n \geq 3$ and $\tilde{g}$ an ambient metric. We shall play with the following three operators:

$$
\begin{array}{lll}
x: \tilde{\mathscr{S}}(w) \rightarrow \tilde{\mathscr{S}}(w+2), & \tilde{\sigma} \mapsto \frac{1}{4} r \tilde{\sigma}, \\
y: \tilde{\mathscr{S}}(w) \rightarrow \tilde{\mathscr{S}}(w-2), & \tilde{\sigma} \mapsto \tilde{\Delta}_{\mathrm{L}} \tilde{\sigma}, \\
h: \tilde{\mathscr{Y}}(w) \rightarrow \tilde{\mathscr{S}}(w), & \tilde{\sigma} \mapsto\left(\tilde{\nabla}_{T}+\frac{n+2}{2}\right) \tilde{\sigma}=\left(w+\frac{n}{2}-1\right) \tilde{\sigma} .
\end{array}
$$

Just as in the case of the classical GJMS construction, one can verify the following.

Proposition 2.1. The operators $x, y$, h enjoy the $\mathfrak{s l}(2)$ commutation relations:

$$
[h, x]=2 x, \quad[h, y]=-2 y, \quad[x, y]=h .
$$


The proof is left to the reader. Consequently we have the following identities:

$$
\begin{aligned}
{\left[y^{m}, x\right] } & =-m y^{m-1}(h-m+1), \\
{\left[x^{m}, y\right] } & =m x^{m-1}(h+m-1), \\
y^{m-1} x^{m-1} & =(-1)^{m-1}(m-1) ! h(h+1) \cdots(h+m-2)+x Z_{m},
\end{aligned}
$$

where $Z_{m}$ is some polynomial in $x, y, h$.

We are going to verify that $x, y$, and $h$ preserve the subspaces $\tilde{\mathscr{Y}}_{\mathrm{TT}}^{X}(w)$ when $n$ is odd and $\tilde{\mathscr{S}}_{\text {aTT }}^{X}(w)$ when $n$ is even. For this we need two lemmas.

Lemma 2.2. For $\tilde{f} \in \tilde{\mathscr{E}}(w), \tilde{\tau} \in \tilde{T}(w)$,

$$
\begin{gathered}
\tilde{f}=O\left(r^{m}\right) \Longrightarrow \tilde{\Delta} \tilde{f}=O\left(r^{m-1}\right), \\
\tilde{\tau}=O^{-}\left(r^{m}\right) \Longrightarrow \tilde{\Delta} \tilde{\tau}=O^{-}\left(r^{m-1}\right) .
\end{gathered}
$$

In (2-5), we may also replace $\tilde{\Delta}$ with the Hodge Laplacian $\tilde{\Delta}_{\mathrm{H}}$.

Proof. First we compute $\tilde{\Delta}\left(r^{m}\right)$ :

$$
\tilde{\Delta}\left(r^{m}\right)=-\tilde{\nabla}^{I} \tilde{\nabla}_{I}\left(r^{m}\right)=-\tilde{\nabla}^{I}\left(2 m r^{m-1} T_{I}\right)=-2 m(2 m+n) r^{m-1} .
$$

Hence it is clear that $\tilde{f}=O\left(r^{m}\right)$ implies $\tilde{\Delta} \tilde{f}=O\left(r^{m-1}\right)$ and that $\tilde{\tau}=O\left(r^{m}\right)$ implies $\tilde{\Delta} \tilde{\tau}=O\left(r^{m-1}\right)$. So, to prove (2-5), it remains to show that $\tilde{\Delta}\left(r^{m-1} \tilde{f} T_{I}\right)$ is $O^{-}\left(r^{m-1}\right)$. This is checked directly:

$$
\tilde{\nabla}_{J}\left(r^{m-1} \tilde{f} T_{I}\right)=2(m-1) r^{m-2} \tilde{f} T_{I} T_{J}+r^{m-1} \tilde{f} \tilde{g}_{I J}+r^{m-1} T_{I} \tilde{\nabla}_{J} \tilde{f}
$$

and therefore

$$
\tilde{\Delta}\left(r^{m-1} \tilde{f} T_{I}\right)=-2(m-1)(2 m+n+2 w) r^{m-2} \tilde{f} T_{I}+O\left(r^{m-1}\right) .
$$

By Bochner's formula $\tilde{\Delta}_{\mathrm{H}} \tilde{\tau}_{I}=\tilde{\Delta} \tilde{\tau}_{I}+\widetilde{\operatorname{Ric}}_{I}^{J} \tilde{\tau}_{J}, \tilde{\Delta}_{\mathrm{H}} \tilde{\tau}=O^{-}\left(r^{m-1}\right)$ is clear.

Let $(D \widetilde{R i c})^{\circ}: \tilde{\mathscr{T}}(w) \rightarrow \tilde{\mathscr{T}}(w-4)$ be defined by

$$
\left((D \widetilde{\mathrm{Ric}})^{\circ} \tilde{\sigma}\right)_{I}=\left(\tilde{\nabla}_{I} \widetilde{\operatorname{Ric}}_{J K}-\tilde{\nabla}_{J}{\widetilde{\operatorname{Ric}_{I K}}}-\tilde{\nabla}_{K}{\widetilde{\operatorname{Ric}_{I J}}}_{I}\right) \tilde{\sigma}^{J K}
$$

Then it is known that, on any symmetric 2-tensor,

$$
\delta_{\tilde{g}} \circ \tilde{\Delta}_{\mathrm{L}}=\tilde{\Delta}_{\mathrm{H}} \circ \delta_{\tilde{g}}+(\text { D } \widetilde{\text { Ric }})^{\circ} .
$$

Lemma 2.3. When $n$ is even and $2-n \leq w \leq 2$,

$$
\tilde{\sigma} \in \tilde{\mathscr{T}}_{\mathrm{aTT}}^{X}(w) \Longrightarrow(D \widetilde{\mathrm{Ric}})^{\circ} \tilde{\sigma}=O^{-}\left(r^{n / 2-1}\right) .
$$


Proof. Let $\widetilde{\mathrm{Ric}}=r^{n / 2-1} \tilde{S}$. Then

$$
\tilde{\nabla}_{I} \widetilde{\operatorname{Ric}}_{J K}=(n-2) r^{n / 2-2} T_{I} \tilde{S}_{J K}+O\left(r^{n / 2-1}\right) .
$$

Therefore

$$
\left(\tilde{\nabla}_{I} \widetilde{\operatorname{Ric}}_{J K}\right) \tilde{\sigma}^{J K}=(n-2) r^{n / 2-2}\langle\tilde{S}, \tilde{\sigma}\rangle_{\tilde{g}} T_{I}+O\left(r^{n / 2-1}\right) .
$$

On the other hand, since $T\lrcorner \tilde{\sigma}$ is at least $O^{-}(r)$, we can write $T^{I} \tilde{\sigma}_{I J}=\tilde{f} T_{J}+O(r)$. Hence, by (2-7) and (1-4),

$$
\left(\tilde{\nabla}_{J} \widetilde{\operatorname{Ric}}_{I K}\right) \tilde{\sigma}^{J K}=(n-2) r^{n / 2-2} \tilde{f} T^{K} \tilde{S}_{I K}+O\left(r^{n / 2-1}\right)=O\left(r^{n / 2-1}\right) .
$$

Consequently, $(\widetilde{D \text { Ric }})^{\circ} \tilde{\sigma}=O^{-}\left(r^{n / 2-1}\right)$.

Proposition 2.4. If $n$ is odd, then, for any $w$,

$x\left(\tilde{\mathscr{Y}}_{\mathrm{TT}}^{X}(w)\right) \subset \tilde{\mathscr{Y}}_{\mathrm{TT}}^{X}(w+2), \quad y\left(\tilde{\mathscr{Y}}_{\mathrm{TT}}^{X}(w)\right) \subset \tilde{\mathscr{Y}}_{\mathrm{TT}}^{X}(w-2), \quad h\left(\tilde{\mathscr{Y}}_{\mathrm{TT}}^{X}(w)\right) \subset \tilde{\mathscr{Y}}_{\mathrm{TT}}^{X}(w)$.

If $n$ is even,

$$
\begin{array}{lr}
x\left(\tilde{\mathscr{S}}_{\mathrm{aTT}}^{X}(w)\right) \subset \tilde{\mathscr{S}}_{\mathrm{aTT}}^{X}(w+2), & 2-n \leq w \leq 0, \\
y\left(\tilde{\mathscr{S}}_{\mathrm{aTT}}^{X}(w)\right) \subset \tilde{\mathscr{S}}_{\mathrm{aTT}}^{X}(w-2), & -n \leq w \leq 2, \\
h\left(\tilde{\mathscr{S}}_{\mathrm{aTT}}^{X}(w)\right) \subset \tilde{\mathscr{S}}_{\mathrm{aTT}}^{X}(w), & 2-n \leq w \leq 2 .
\end{array}
$$

Proof. Since the case $n$ odd is easier to prove, we discuss the case $n$ even. It is clear that $h\left(\tilde{\mathscr{Y}}_{\mathrm{aTT}}^{X}(w)\right) \subset \tilde{\mathscr{Y}}_{\mathrm{aTT}}^{X}(w)$. For $\tilde{\sigma} \in \tilde{\mathscr{Y}}_{\mathrm{aTT}}^{X}(w)$, we have $\left.\left.T\right\lrcorner(r \tilde{\sigma})=r T\right\lrcorner \tilde{\sigma}=$ $O^{-}\left(r^{\lceil(n-2+w) / 2\rceil+2}\right), \operatorname{tr}_{\tilde{g}}(r \tilde{\sigma})=r \operatorname{tr}_{\tilde{g}} \tilde{\sigma}=O\left(r^{\lceil(n-2+w) / 2\rceil+1}\right)$, and

$$
\left.\delta_{\tilde{g}}(r \tilde{\sigma})=-2 T\right\lrcorner \tilde{\sigma}+r \delta_{\tilde{g}} \tilde{\sigma}=O^{-}\left(r^{\lceil(n-2+w) / 2\rceil+1}\right) .
$$

Hence $x \tilde{\sigma} \in \tilde{\mathscr{Y}}_{\mathrm{aTT}}^{X}(w+2)$. It remains to show that $y \tilde{\sigma} \in \tilde{\mathscr{Y}}_{\mathrm{aTT}}^{X}(w-2)$. The trace of $\tilde{\Delta}_{\mathrm{L}} \tilde{\sigma}$ is $\operatorname{tr}_{\tilde{g}} \tilde{\Delta}_{\mathrm{L}} \tilde{\sigma}=\tilde{\Delta}\left(\operatorname{tr}_{\tilde{g}} \tilde{\sigma}\right)=O\left(r^{\lceil(n-2+w) / 2\rceil-1}\right)$ by (2-4). Furthermore,

$$
\tilde{\nabla}_{K}\left(T^{J} \tilde{\sigma}_{I J}\right)=\delta_{K}^{J} \tilde{\sigma}_{I J}+T^{J} \tilde{\nabla}_{K} \tilde{\sigma}_{I J}=\tilde{\sigma}_{I K}+T^{J} \tilde{\nabla}_{K} \tilde{\sigma}_{I J}
$$

and hence

$$
\begin{aligned}
\tilde{\Delta}\left(T^{J} \tilde{\sigma}_{I J}\right) & =-\tilde{\nabla}^{K} \tilde{\sigma}_{I K}-\tilde{\nabla}^{K}\left(T^{J} \tilde{\nabla}_{K} \tilde{\sigma}_{I J}\right) \\
& =-2 \tilde{\nabla}^{K} \tilde{\sigma}_{I K}-T^{J} \tilde{\nabla}^{K} \tilde{\nabla}_{K} \tilde{\sigma}_{I J}=-2 \tilde{\nabla}^{K} \tilde{\sigma}_{I K}+T^{J} \tilde{\Delta}_{\mathrm{L}} \tilde{\sigma}_{I J} ;
\end{aligned}
$$

the last equality is because of (1-4). This implies $T\lrcorner \tilde{\Delta}_{\mathrm{L}} \tilde{\sigma}=O^{-}\left(r^{\lceil(n-2+w) / 2\rceil}\right)$. Finally, (2-6) and Lemma 2.3 show $\delta_{\tilde{g}} \tilde{\Delta}_{\mathrm{L}} \tilde{\sigma}=O^{-}\left(r^{\lceil(n-2+w) / 2\rceil-1}\right)$.

Theorem 2.5. Let $k \in \mathbb{Z}_{+}$if $n$ is odd, and $k \in\{1,2, \ldots, n / 2\}$ if $n$ is even. For any $\varphi \in \mathscr{Y}_{0}(-n / 2+2+k)$, let $\tilde{\sigma} \in \tilde{\mathscr{S}}(-n / 2+2+k)$ be any extension of the ambient lift $\tilde{\varphi}$. Then $\tilde{\Delta}_{\mathrm{L}}^{k} \tilde{\sigma} \mid \mathrm{g}$ depends only on $\varphi$ and not on the extension. Furthermore, $\left.\tilde{\Delta}_{\mathrm{L}}^{k} \tilde{\sigma}\right|_{T M}$ makes sense as a section in $\mathscr{Y}(-n / 2+2-k)$. 
Proof. We work on the case $n$ even only. Any two extensions of $\tilde{\varphi}$ differ by a tensor of the form $r \tilde{\tau}$, where $\tilde{\tau} \in \tilde{\mathscr{Y}}_{0}(-n / 2+k)$. Equation (2-1) shows that the commutator $\left[\tilde{\Delta}_{\mathrm{L}}^{k}, r\right]$ vanishes on $\tilde{\mathscr{S}}_{0}(-n / 2+k)$ and hence $\tilde{\Delta}_{\mathrm{L}}^{k}(r \tilde{\tau}) \mid \varphi=0$. In particular, using Lemma 1.2 one can take $\tilde{\sigma} \in \tilde{\mathscr{T}}_{\mathrm{aTT}}^{X}(-n / 2+2+k)$ as an extension of $\tilde{\varphi}$. Then by Proposition 2.4, $\tilde{\Delta}_{\mathrm{L}}^{k} \tilde{\sigma} \in \tilde{\mathscr{T}}_{\mathrm{aTT}}^{X}(-n / 2+2-k)$ and $\left.\tilde{\Delta}_{\mathrm{L}}^{k} \tilde{\sigma}\right|_{T M}$ is defined.

Theorem 2.6. Let $k \in \mathbb{Z}_{+}$if $n$ is odd, and $k \in\{1,2, \ldots, n / 2\}$ if $n$ is even. Let $\varphi \in \mathscr{Y}_{0}(-n / 2+2+k)$ and let $\tilde{\varphi}$ be its ambient lift. Then there exists a solution $\tilde{\sigma} \in \tilde{\mathscr{Y}}_{\mathrm{TT}}^{X}(-n / 2+2+k)$ if $n$ is odd, and $\tilde{\sigma} \in \tilde{\mathscr{Y}}_{\mathrm{aTT}}^{X}(-n / 2+2+k)$ if $n$ is even, to the problem

$$
\tilde{\Delta}_{\mathrm{L}} \tilde{\sigma}=O\left(r^{k-1}\right),\left.\quad \tilde{\sigma}\right|_{\varphi}=\tilde{\varphi},
$$

which is unique modulo $O\left(r^{k}\right)$. For any such $\tilde{\sigma},\left.\left(r^{1-k} \tilde{\Delta}_{\mathrm{L}} \tilde{\sigma}\right)\right|_{\varphi}$ is independent of the ambiguity that lives in $\tilde{\sigma}$, and agrees with $\tilde{\Delta}_{\mathrm{L}}^{k} \tilde{\sigma} \mid \mathrm{g}$ up to a constant factor:

$$
\left.\left(r^{1-k} \tilde{\Delta}_{\mathrm{L}} \tilde{\sigma}\right)\right|_{\varphi}=\left.\frac{1}{4^{k-1}(k-1) !^{2}} \tilde{\Delta}_{\mathrm{L}}^{k} \tilde{\sigma}\right|_{\varphi} .
$$

Proof. We work on the case $n$ even only. Let us begin with an arbitrary extension $\tilde{\sigma}_{(0)} \in \tilde{\mathscr{Y}}_{\mathrm{aTT}}^{X}(-n / 2+2+k)$ of $\tilde{\varphi}$. If an extension $\tilde{\sigma}_{(m-1)}$ satisfies $\tilde{\Delta}_{\mathrm{L}} \tilde{\sigma}_{(m-1)}=O\left(r^{m-1}\right)$, then it has a modification $\tilde{\sigma}_{(m)}=\tilde{\sigma}_{(m-1)}+r^{m} \tilde{\sigma}_{1}, \tilde{\sigma}_{1} \in \tilde{\mathscr{T}}_{\text {aTT }}^{X}(-n / 2+2+k-2 m)$, which is unique modulo $O\left(r^{m+1}\right)$, satisfying $\tilde{\Delta}_{\mathrm{L}} \tilde{\sigma}_{(m)}=O\left(r^{m}\right)$. In fact, by (2-2), we have

$$
\tilde{\Delta}_{\mathrm{L}}\left(r^{m} \tilde{\sigma}_{1}\right)=4 m r^{m-1}(m-k) \tilde{\sigma}_{1}+r^{m} \tilde{\Delta}_{\mathrm{L}} \tilde{\sigma}_{1} .
$$

Thus $\tilde{\sigma}_{1}$ can be taken so that $\tilde{\Delta}_{\mathrm{L}} \tilde{\sigma}_{(m)}=O\left(r^{m}\right)$ unless $m=k$. Hence there is a $\tilde{\sigma}$ with the property stated in the theorem. Let $\tilde{\Delta}_{\mathrm{L}} \tilde{\sigma}=r^{k-1} \tilde{F}$, with $\tilde{F} \in \tilde{\mathscr{T}}_{\mathrm{aTT}}^{X}(-n / 2+2-k)$. Then, by (2-3), $\tilde{\Delta}_{\mathrm{L}}^{k} \tilde{\sigma}=4^{k-1} y^{k-1} x^{k-1} \tilde{F}=4^{k-1}(k-1) !^{2} \tilde{F}+O(r)$. Hence (2-9).

Except in the case where $n$ is even and $k=n / 2,\left.\left(\tilde{\Delta}_{\mathrm{L}}^{k} \tilde{\sigma}\right)\right|_{T M}$ is trace-free since $\operatorname{tr}_{\tilde{g}} \tilde{\Delta}_{\mathrm{L}}^{k} \tilde{\sigma}$ and $\left.T\right\lrcorner \tilde{\Delta}_{\mathrm{L}}^{k} \tilde{\sigma}$ are both $O(r)$.

Definition 2.7. Let $(M,[g])$ be a conformal manifold of dimension $n \geq 3$ and $\tilde{g}$ an ambient metric. We call

$$
P_{k}: \mathscr{Y}_{0}(-n / 2+2+k) \rightarrow \mathscr{Y}_{0}(-n / 2+2-k), \quad P_{k} \varphi=\operatorname{tf}_{g}\left(\left.\tilde{\Delta}_{\mathrm{L}}^{k} \tilde{\sigma}\right|_{T M}\right)
$$

the GJMS operator on trace-free symmetric 2-tensors, where $\tilde{\sigma} \in \tilde{\mathscr{T}}(-n / 2+2+k)$ is any extension of the ambient lift of $\varphi$. (One can remove $\mathrm{tf}_{g}$ unless $n$ is even and $k=n / 2$.) In particular, when $n=\operatorname{dim} M \geq 4$ is even,

$$
P=P_{n / 2}: \mathscr{Y}_{0}(2) \rightarrow \mathscr{S}_{0}(2-n)
$$

is called the critical GJMS operator on trace-free symmetric 2-tensors. 
Theorem 2.8. The GJMS operators on trace-free symmetric 2-tensors do not depend on the choice of $\tilde{g}$, and hence are conformally invariant differential operators.

For the case where $n$ is even and $k=n / 2$, the direct verification of the conformal invariance is not easy. We will see in Theorem 3.4 that, up to a constant factor, $P \varphi$ is equal to $\operatorname{tf}_{g} \mathrm{O}_{g}^{\prime} \varphi$, which is clearly conformally invariant. Here, we prove the theorem in the case $n$ odd and the case $n$ even, $k \leq n / 2-1$.

Proof of Theorem 2.8 except the case where $(n, k)=($ even, $n / 2)$. By Theorem 2.6, we may work with $r^{1-k} \tilde{\Delta}_{\mathrm{L}} \tilde{\sigma}$ instead of $\tilde{\Delta}_{\mathrm{L}}^{k} \tilde{\sigma}$. Let $\tilde{g}$ be an ambient metric, $\varphi \in$ $\mathscr{Y}_{0}(-n / 2+2+k)$ and, let $\tilde{\sigma}$ be a solution to the problem stated in Theorem 2.6. Then, if $\Phi$ is an ambient-equivalence map, $\Phi^{*} \tilde{\sigma}$ solves the same problem with respect to $\Phi^{*} \tilde{g}$. Since $\left(\Phi^{*} r\right)^{1-k} \tilde{\Delta}_{\mathrm{L}, \Phi^{*} \tilde{g}}\left(\Phi^{*} \tilde{\sigma}\right)=\Phi^{*}\left(r^{1-k} \tilde{\Delta}_{\mathrm{L}} \sigma\right)$, the restrictions of $\left(\Phi^{*} r\right)^{1-k} \tilde{\Delta}_{\mathrm{L}, \Phi^{*} \tilde{g}}\left(\Phi^{*} \tilde{\sigma}\right)$ and $r^{1-k} \tilde{\Delta}_{\mathrm{L}} \sigma$ to $T M$ coincide. Therefore we may assume that $\tilde{g}$ is in normal form.

When $n$ is odd, the assertion is now clear because $\tilde{g}$ is formally unique if it is in normal form. So we assume that $n$ is even in what follows. It suffices to show that, if $\tilde{g}, \hat{\tilde{g}}$ are ambient metrics in normal form and $\tilde{\sigma} \in \tilde{\mathscr{T}}_{\mathrm{aTT}}^{X}(-n / 2+2+k)$,

$$
\hat{\tilde{\Delta}}_{\mathrm{L}} \tilde{\sigma}-\tilde{\Delta}_{\mathrm{L}} \tilde{\sigma}=O\left(\rho^{n / 2-2}\right) \quad \text { and } \quad \hat{\tilde{\Delta}}_{\mathrm{L}} \tilde{\sigma}_{i j}-\tilde{\Delta}_{\mathrm{L}} \tilde{\sigma}_{i j}=O\left(\rho^{n / 2-1}\right) .
$$

Let $D^{K}{ }_{I J}=\hat{\tilde{\Gamma}}_{I J}^{K}-\tilde{\Gamma}_{I J}^{K}$. From [Fefferman and Graham 2012, Equation (3.16)], one concludes that $D^{K}{ }_{I J}=O\left(\rho^{n / 2-1}\right)$ and $\tilde{\nabla}^{I} D^{K}{ }_{I J}=O\left(\rho^{n / 2-1}\right)$. Therefore

$$
\hat{\tilde{\Delta}} \tilde{\sigma}_{I J}-\tilde{\Delta} \tilde{\sigma}_{I J}=\tilde{\nabla}^{K}\left(2 D_{K(I}^{L} \tilde{\sigma}_{J) L}\right)+O\left(\rho^{n / 2-1}\right)=O\left(\rho^{n / 2-1}\right) .
$$

In addition, $\widehat{\operatorname{Ric}}=\widetilde{\operatorname{Ric}}+O\left(\rho^{n / 2-1}\right)$ and $\hat{\tilde{R}}=\tilde{R}+O\left(\rho^{n / 2-2}\right)$ by [ibid., Equation (6.1)]. Hence $\hat{\tilde{\Delta}}_{\mathrm{L}} \tilde{\sigma}-\tilde{\Delta}_{\mathrm{L}} \tilde{\sigma}=O\left(\rho^{n / 2-2}\right)$. Moreover, if $\tilde{S}_{I J K L}=\hat{\tilde{R}}_{I J K L}-\tilde{R}_{I J K L}$, then

$$
\begin{aligned}
\hat{\tilde{\Delta}}_{\mathrm{L}} \tilde{\sigma}_{i j}-\tilde{\Delta}_{\mathrm{L}} \tilde{\sigma}_{i j} \\
=-2 t^{-4}\left(g_{\rho}^{-1}\right)^{k m}\left(g_{\rho}^{-1}\right)^{l n} \tilde{S}_{i k j l} \tilde{\sigma}_{m n}-4 t^{-3}\left(g_{\rho}^{-1}\right)^{k m} \tilde{S}_{i k j \infty} \tilde{\sigma}_{m 0}+4 t^{-4} \rho\left(g_{\rho}^{-1}\right)^{k m} \tilde{S}_{i k j \infty} \tilde{\sigma}_{m \infty} \\
\quad-2 t^{-2} \tilde{S}_{i \infty j \infty} \tilde{\sigma}_{00}+4 t^{-3} \rho \tilde{S}_{i \infty j \infty} \tilde{\sigma}_{0 \infty}-8 t^{-4} \rho^{2} \tilde{S}_{i \infty j \infty} \tilde{\sigma}_{\infty \infty}+O\left(\rho^{n / 2-1}\right) .
\end{aligned}
$$

Again by [ibid., Equation (6.1)], we have $\tilde{S}_{i j k l}=O\left(\rho^{n / 2-1}\right), \tilde{S}_{i j k \infty}=O\left(\rho^{n / 2-1}\right)$, $\tilde{S}_{i \infty k \infty}=O\left(\rho^{n / 2-2}\right)$ and hence $\tilde{\tilde{\Delta}}_{\mathrm{L}} \tilde{\sigma}_{i j}-\tilde{\Delta}_{\mathrm{L}} \tilde{\sigma}_{i j}=O\left(\rho^{n / 2-1}\right)$.

We close this section with a lemma that is proved just like the construction of $\tilde{\sigma}$ in Theorem 2.6.

Lemma 2.9. Let $k \in \mathbb{Z}_{+}$. For any $\tilde{f}_{1} \in \tilde{\mathscr{E}}(-n / 2-2+k)$, there exists $\tilde{f} \in \tilde{\mathscr{E}}(-n / 2+k)$ such that

$$
\tilde{\Delta} \tilde{f}=\tilde{f}_{1}+O\left(r^{k-1}\right) .
$$


Likewise, for any $\tilde{\tau}_{1} \in \tilde{\mathscr{T}}(-n / 2-1+k)$, there exists $\tilde{\tau} \in \tilde{\mathscr{T}}(-n / 2+1+k)$ such that

$$
\tilde{\Delta} \tilde{\tau}=\tilde{\tau}_{1}+O\left(r^{k-1}\right) .
$$

In both problems, we may arbitrarily prescribe the values along $\mathscr{G}$; if we prescribe $\tilde{f}|\varphi, \tilde{\tau}| \varphi$, then $\tilde{f}, \tilde{\tau}$ are unique modulo $O\left(r^{k}\right)$.

\section{The variations of obstruction tensor and $Q$-curvature}

Let $\tilde{g}$ be an ambient metric for a conformal manifold $(M,[g])$ of dimension $n \geq 3$. Recall that, from general calculations on (pseudo-)Riemannian curvature tensors, the differential of the Ricci tensor operator (which we write as Ric here) is

$$
\operatorname{Ric}_{\tilde{g}}^{\prime} \tilde{\sigma}=\frac{1}{2} \tilde{\Delta}_{\mathrm{L}} \tilde{\sigma}-\delta_{\tilde{g}}^{*} \mathscr{B}_{\tilde{g}} \tilde{\sigma}
$$

where $\delta_{\tilde{g}}^{*}$ is the dual of the divergence $\left(\delta_{\tilde{g}}^{*} \tilde{\tau}\right)_{I J}=\tilde{\nabla}_{(I} \tilde{\tau}_{J)}$ and $\mathscr{B}_{\tilde{g}}$ is defined by $\mathscr{B}_{\tilde{g}} \tilde{\sigma}=\delta_{\tilde{g}} \tilde{\sigma}+\frac{1}{2} d\left(\operatorname{tr}_{\tilde{g}} \tilde{\sigma}\right)$. Therefore, for $n$ even, a solution $\tilde{\sigma} \in \tilde{\mathscr{Y}}_{\mathrm{aTT}}^{X}(2)$ to the problem in Theorem 2.6 approximately solves $\operatorname{Ric}_{\tilde{g}}^{\prime} \tilde{\sigma}=0$, and hence it is expected that we can read off $\mathrm{O}_{g}^{\prime} \varphi$ from the asymptotics of $\tilde{\sigma}$. This will finally turn out to be true, but since the definition of $\mathbb{O}$ depends on the existence theorem of normal-form ambient metrics, in order to capture $\mathrm{O}_{g}^{\prime} \varphi$ our starting point has to be infinitesimal modifications of ambient metrics in normal form. The differential equation that they (approximately) satisfy is different from $\tilde{\Delta}_{\mathrm{L}} \tilde{\sigma}=0$. So we shall establish a method for translating solutions of the two equations.

Let $(M,[g])$ be an $n$-dimensional conformal manifold with $n \geq 4$ even and $\varphi \in \mathscr{Y}_{0}(2)$. Suppose that $\boldsymbol{g}_{s}$ is a family of conformal metrics (here we use $s$ for the parameter, because $t$ will denote a coordinate on $\tilde{\mathscr{G}}$ ) with $\boldsymbol{g}_{0}=\boldsymbol{g}$ such that $\left.\dot{\boldsymbol{g}}_{s}\right|_{s=0}=\varphi$. Let $g \in[g]$ be any representative metric, and $g_{s}$ the corresponding representatives of $\boldsymbol{g}_{s}$. By the method of [Fefferman and Graham 2012], we can construct a family of ambient metrics

$$
\tilde{g}_{s}=2 \rho d t^{2}+2 t d t d \rho+t^{2} g_{\rho}^{s}
$$

such that $g_{0}^{s}=g_{s}$ and $g_{\rho}^{s}$ smoothly depends on the two variables $\rho, s$. All these metrics satisfy $\widetilde{\operatorname{Ric}}_{s}=O\left(r^{n / 2-1}\right)$ and $\left.T\right\lrcorner \widetilde{\operatorname{Ric}}_{s}=O^{-}\left(r^{n / 2}\right)$. Differentiating these equations, we conclude that $\tilde{\sigma}=\tilde{\sigma}_{\text {norm }}=\left.(d / d s) \tilde{g}_{s}\right|_{s=0}$ solves

$$
\left.\operatorname{Ric}_{\tilde{g}}^{\prime} \tilde{\sigma}=O\left(r^{n / 2-1}\right), \quad T\right\lrcorner \operatorname{Ric}_{\tilde{g}}^{\prime} \tilde{\sigma}=O^{-}\left(r^{n / 2}\right) .
$$

Note that it satisfies $T\lrcorner \tilde{\sigma}_{\text {norm }}=0, \operatorname{tr}_{\tilde{g}} \tilde{\sigma}_{\text {norm }}=O(r)$, and hence

$$
T^{I} \tilde{\nabla}^{J}\left(\tilde{\sigma}_{\mathrm{norm}}\right)_{I J}=\tilde{\nabla}^{J}\left(T^{I}\left(\tilde{\sigma}_{\mathrm{norm}}\right)_{I J}\right)-\tilde{g}^{I J}\left(\tilde{\sigma}_{\mathrm{norm}}\right)_{I J}=O(r) ;
$$


therefore it holds that

$$
\left.T\lrcorner \mathscr{B}_{\tilde{g}} \tilde{\sigma}_{\text {norm }}=T\right\lrcorner \delta_{\tilde{g}} \tilde{\sigma}_{\text {norm }}+\frac{1}{2} T\left(\operatorname{tr}_{\tilde{g}} \tilde{\sigma}_{\text {norm }}\right)=O(r) .
$$

Since the obstruction tensor $\mathrm{O}=\mathrm{O}_{s}$ is defined by

$$
\mathrm{O}_{s}=\left.c_{n}\left(r^{1-n / 2} \widetilde{\operatorname{Ric}}_{S}\right)\right|_{T M}, \quad c_{n}=(-1)^{n / 2-1} \frac{2^{n-2}(n / 2-1) !^{2}}{n-2},
$$

we have

$$
\mathrm{O}_{\mathbf{g}}^{\prime} \varphi=\left.c_{n}\left(r^{1-n / 2} \widetilde{\operatorname{Ric}}_{\tilde{g}}^{\prime} \tilde{\sigma}_{\text {norm }}\right)\right|_{T M}
$$

Lemma 3.1. Let $\tilde{\sigma}_{\text {norm }}$ be as above. Then, there exists a dilation-invariant vector field $\tilde{\xi}$ on $\tilde{\varphi}$ such that $\left.\tilde{\xi}\right|_{\varphi}=0$ and

$$
\mathscr{B}_{\tilde{g}}\left(\tilde{\sigma}_{\text {norm }}+\mathscr{K}_{\tilde{g}} \tilde{\xi}\right)=O\left(r^{n / 2}\right),
$$

where $\mathscr{K}_{\tilde{g}}$ is the Killing operator: $\left(\mathscr{K}_{\tilde{g}} \tilde{\xi}\right)_{I J}=2 \tilde{\nabla}_{(I} \tilde{\xi}_{J)}$. Such a $\tilde{\xi}$ is unique modulo $O\left(r^{n / 2+1}\right)$ and satisfies $\tilde{g}(T, \tilde{\xi})=O\left(r^{2}\right), \operatorname{tr}_{\tilde{g}} \mathcal{K}_{\tilde{g}} \tilde{\xi}=O(r)$.

Proof. The equation to be solved is $\mathscr{R}_{\tilde{g}} \mathscr{K}_{\tilde{g}} \tilde{\xi}=-\mathscr{T}_{\tilde{g}} \tilde{\sigma}_{\text {norm }}+O\left(r^{n / 2}\right)$. By a straightforward calculation,

$$
\left(\mathscr{B}_{\tilde{g}} \mathscr{K}_{\tilde{g}} \tilde{\xi}\right)_{I}=\tilde{\Delta} \tilde{\xi}_{I}-\widetilde{\operatorname{Ric}}_{I J} \tilde{\xi}^{J}
$$

Since $\widetilde{\operatorname{Ric}}_{I J} \tilde{\xi}^{J}=O\left(r^{n / 2}\right)$ for any $\tilde{\xi}$ satisfying $\left.\tilde{\xi}\right|_{\varphi}=0$, the equation simplifies to $\tilde{\Delta} \tilde{\xi}=-\mathscr{B}_{\tilde{g}} \tilde{\sigma}_{\text {norm }}+O\left(r^{n / 2}\right)$. By Lemma $2.9, \tilde{\xi}$ is uniquely determined up to an $O\left(r^{n / 2+1}\right)$ ambiguity.

If we write $\tilde{\xi}=r V$, then $\tilde{\Delta} \tilde{\xi}=-2 n V+O(r)$. On the other hand, $T\lrcorner \tilde{\Delta} \tilde{\xi}=$ $-2 T\lrcorner \mathscr{P}_{\tilde{g}} \tilde{\sigma}_{\text {norm }}+O\left(r^{n / 2}\right)$ should be $O(r)$ by (3-2). Consequently $\left.T\right\lrcorner V=O(r)$, i.e., $T\lrcorner \tilde{\xi}=O\left(r^{2}\right)$. Moreover, $\operatorname{tr}_{\tilde{g}} \mathscr{K}_{\tilde{g}} \tilde{\xi}=2 \tilde{\nabla}^{I} \tilde{\xi}_{I}=4 T^{I} V_{I}+O(r)=O(r)$.

Let $\tilde{\sigma}=\tilde{\sigma}_{\text {norm }}+\mathscr{K}_{\tilde{g}} \tilde{\xi} \in \tilde{\mathscr{S}}(2)$. It is a consequence of the fact that the Ricci operator commutes with diffeomorphisms that $\operatorname{Ric}_{\tilde{g}}^{\prime} \mathscr{K}_{\tilde{g}} \tilde{\xi}=\operatorname{Ric}_{\tilde{g}}^{\prime} \mathscr{L}_{\tilde{\xi}} \tilde{g}=\mathscr{L}_{\tilde{\xi}} \widetilde{\text { Ric. }}$.


and $T\lrcorner \mathscr{L}_{\tilde{\xi}} \widetilde{\operatorname{Ric}}=O^{-}\left(r^{n / 2}\right)$. Therefore $\left.\operatorname{Ric}_{\tilde{g}}^{\prime} \tilde{\sigma}=O\left(r^{n / 2-1}\right), T\right\lrcorner \operatorname{Ric}_{\tilde{g}}^{\prime} \tilde{\sigma}=O^{-}\left(r^{n / 2}\right)$. Moreover, $\mathscr{B}_{\tilde{g}} \tilde{\sigma}=O\left(r^{n / 2}\right)$ and hence $\left.\delta_{\tilde{g}}^{*} \mathscr{P}_{\tilde{g}} \tilde{\sigma}=O\left(r^{n / 2-1}\right), T\right\lrcorner \delta_{\tilde{g}}^{*} \mathscr{P}_{\tilde{g}} \tilde{\sigma}=O^{-}\left(r^{n / 2}\right)$. Thus we conclude

$$
\left.\tilde{\Delta}_{\mathrm{L}} \tilde{\sigma}=O\left(r^{n / 2-1}\right), \quad T\right\lrcorner \tilde{\Delta}_{\mathrm{L}} \tilde{\sigma}=O^{-}\left(r^{n / 2}\right) .
$$

Lemma 3.2. Let $\tilde{\sigma}_{\text {norm }}$ and $\tilde{\xi}$ be as in Lemma 3.1. Then $\tilde{\sigma}=\tilde{\sigma}_{\text {norm }}+\mathscr{K}_{\tilde{g}} \tilde{\xi} \in \tilde{\mathscr{Y}}_{\mathrm{aTT}}^{X}(2)$ and it is a solution to (3-3).

Proof. It remains to show that $\tilde{\sigma} \in \tilde{\mathscr{Y}}_{\mathrm{aTT}}^{X}(2)$. By taking the trace of (3-3), we obtain $\tilde{\Delta}\left(\operatorname{tr}_{\tilde{g}} \tilde{\sigma}\right)=O\left(r^{n / 2-1}\right)$. In addition, since $\operatorname{tr}_{\tilde{g}} \mathcal{K}_{\tilde{g}} \tilde{\xi}=O(r)$, we have $\left.\left(\operatorname{tr}_{\tilde{g}} \tilde{\sigma}\right)\right|_{\varphi}=0$. 
Hence, by Lemma 2.9, $\operatorname{tr}_{\tilde{g}} \tilde{\sigma}=O\left(r^{n / 2}\right)$. Then $\mathscr{B}_{\tilde{g}} \tilde{\sigma}=O\left(r^{n / 2}\right)$ implies $\delta_{\tilde{g}} \tilde{\sigma}=$ $O^{-}\left(r^{n / 2}\right)$. Furthermore,

$$
\tilde{\Delta}\left(T^{J} \tilde{\sigma}_{I J}\right)=T^{J} \tilde{\Delta} \tilde{\sigma}_{I J}-2 \tilde{\nabla}^{J} \tilde{\sigma}_{I J}=T^{J} \tilde{\Delta}_{\mathrm{L}} \tilde{\sigma}_{I J}-2 \tilde{\nabla}^{J} \tilde{\sigma}_{I J}=O^{-}\left(r^{n / 2}\right)
$$

and

$$
T^{J} \tilde{\sigma}_{I J}=T^{J}\left(\mathscr{K}_{\tilde{g}} \tilde{\xi}\right)_{I J}=T^{J} \tilde{\nabla}_{I} \tilde{\xi}_{J}+T^{J} \tilde{\nabla}_{J} \tilde{\xi}_{I}=\tilde{\nabla}_{I}\left(T^{J} \tilde{\xi}_{J}\right)=O(r) .
$$

Since $\tilde{\Delta}\left(r^{n / 2} \tilde{f} T_{I}\right)=-2 n r^{n / 2-1} \tilde{f} T_{I}+O\left(r^{n / 2}\right)$ for $\tilde{f} \in \tilde{\mathscr{E}}(-n)$, one can determine $\tilde{f}$ so that $\tilde{\Delta}\left(T^{J} \tilde{\sigma}_{I J}+r^{n / 2} \tilde{f} T_{I}\right)=O\left(r^{n / 2}\right)$. Then $T^{J} \tilde{\sigma}_{I J}+r^{n / 2} \tilde{f} T_{I}$ is still $O(r)$, and hence $T\lrcorner \tilde{\sigma}=O^{-}\left(r^{n / 2+1}\right)$ by Lemma 2.9.

Lemma 3.3. Let $\tilde{\sigma}_{\text {norm }}$ and $\tilde{\xi}$ be as in Lemma 3.1 and set $\tilde{\sigma}=\tilde{\sigma}_{\text {norm }}+\mathscr{K}_{\tilde{g}} \tilde{\xi}$. Then $\tilde{\Delta}_{\mathrm{L}} \tilde{\sigma}-2 \operatorname{Ric}_{\tilde{g}}^{\prime} \tilde{\sigma}_{\text {norm }}=O\left(r^{n / 2-1}\right)$, and $\left.\left(r^{1-n / 2}\left(\tilde{\Delta}_{\mathrm{L}} \tilde{\sigma}-2 \operatorname{Ric}_{\tilde{g}}^{\prime} \tilde{\sigma}_{\text {norm }}\right)\right)\right|_{T^{\varphi}}$ vanishes.

Proof. Recall that

$$
\frac{1}{2} \tilde{\Delta}_{\mathrm{L}} \tilde{\sigma}-\operatorname{Ric}_{\tilde{g}}^{\prime} \tilde{\sigma}_{\text {norm }}=\operatorname{Ric}_{\tilde{g}}^{\prime} \mathscr{K}_{\tilde{g}} \tilde{\xi}-\delta_{\tilde{g}}^{*} \mathscr{B}_{\tilde{g}} \tilde{\sigma}=\mathscr{L}_{\tilde{\xi}} \widetilde{\operatorname{Ric}}-\delta_{\tilde{g}}^{*} \mathscr{B}_{\tilde{g}} \tilde{\sigma} .
$$

Let $\widetilde{\text { Ric }}=r^{n / 2-1} S$ and $\tilde{\xi}=r V$. We proved in Lemma 3.1 that $T^{I} V_{I}=O(r)$. As in the proof of Lemma 1.1, we compute

$$
\left(\mathscr{L}_{\tilde{\xi}} \widetilde{\mathrm{Ric}}\right)_{I J}=\tilde{\xi}^{K} \tilde{\nabla}_{K} \widetilde{\operatorname{Ric}}_{I J}+2 \widetilde{\operatorname{Ric}}_{K(I} \tilde{\nabla}_{J)} \tilde{\xi}^{K}=4 r^{n / 2-1} S_{K(I} T_{J)} V^{K}+O\left(r^{n / 2}\right) .
$$

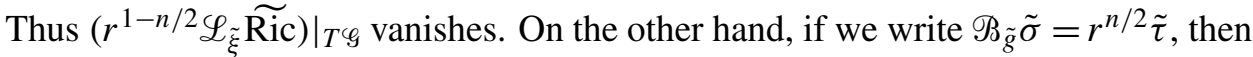

$$
\left(\delta_{\tilde{g}}^{*} \mathscr{S}_{\tilde{g}} \tilde{\sigma}\right)_{I}=\tilde{\nabla}_{(I}\left(r^{n / 2} \tilde{\tau}\right)_{J)}=n r^{n / 2-1} T_{(I} \tilde{\tau}_{J)}+O\left(r^{n / 2}\right),
$$

and hence $\left.\left(r^{1-n / 2} \delta_{\tilde{g}}^{*} \mathscr{S}_{\tilde{g}} \tilde{\sigma}\right)\right|_{T^{\varphi}}=0$. This completes the proof.

Theorem 3.4. Let $(M,[g])$ be a conformal manifold of even dimension $n$. Then the differential of the obstruction tensor ${\mathrm{O}_{g}^{\prime}}_{\mathrm{g}}$ is given by

$$
\mathrm{O}_{\boldsymbol{g}}^{\prime} \varphi=\frac{(-1)^{n / 2-1}}{2(n-2)} P \varphi+\frac{1}{n+2}\langle\mathcal{O}, \varphi\rangle_{\mathbf{g}} \mathbf{g} .
$$

Proof. Let $\tilde{\sigma}_{\text {norm }}, \tilde{\xi}$ as in Lemma 3.1 and $\tilde{\sigma}=\tilde{\sigma}_{\text {norm }}+\mathscr{K}_{\tilde{g}} \tilde{\xi}$. By Lemma 3.2,P $\varphi$ is equal to the trace-free part of $\left.2^{n-2}(n / 2-1) !^{2}\left(r^{1-n / 2} \tilde{\Delta}_{\mathrm{L}} \tilde{\sigma}\right)\right|_{T M}$. By the previous lemma, $\left.\left(r^{1-n / 2} \tilde{\Delta}_{\mathrm{L}} \tilde{\sigma}\right)\right|_{T M}=\left.\left(2 r^{1-n / 2} \operatorname{Ric}_{\tilde{g}}^{\prime} \tilde{\sigma}_{\text {norm }}\right)\right|_{T M}=c_{n}^{-1}{O^{\prime}}_{g}^{\prime} \varphi$. Therefore,

$$
\operatorname{tf}_{g} O_{g}^{\prime} \varphi=\frac{(-1)^{n / 2-1}}{2(n-2)} P \varphi .
$$

On the other hand, $\operatorname{tr}_{g} \mathbb{O}_{g}^{\prime} \varphi=\langle\mathcal{O}, \varphi\rangle_{g}$, for $\operatorname{tr}_{g} \mathbb{O}=0$ for any $g$. Hence (3-4).

Combining the theorem above and (0-4), we obtain the following. 
Corollary 3.5. Let $(M,[g])$ be a compact conformal manifold of even dimension $n \geq 4$ with vanishing obstruction tensor. Let $\boldsymbol{g}_{t}$ be a family of conformal structures such that $\boldsymbol{g}_{0}=\mathbf{g}$. Then the second derivative of the total $Q$-curvature at $t=0$ is

$$
\left.\frac{d^{2}}{d t^{2}} \bar{Q}_{t}\right|_{t=0}=-\frac{1}{4} \int_{M}\langle P \varphi, \varphi\rangle_{g},
$$

where $\varphi=\left.\dot{\boldsymbol{g}}_{t}\right|_{t=0}$ and $P: \mathscr{S}_{0}(2) \rightarrow \mathscr{Y}_{0}(2-n)$ is the critical GJMS operator on trace-free symmetric 2-tensors.

\section{Explicit calculations for conformally Einstein manifolds}

Recall that, for $g \in[g]$ Einstein with $\operatorname{Ric}_{i j}=2 \lambda(n-1) g_{i j}$ so that $P_{i j}=\lambda g_{i j}$, the following formula gives an ambient metric that is genuinely Ricci-flat:

$$
\tilde{g}=2 \rho d t^{2}+2 t d t d \rho+t^{2}(1+\lambda \rho)^{2} g .
$$

The inverse of $\tilde{g}$ is

$$
\left(\tilde{g}^{-1}\right)^{I J}=\left(\begin{array}{ccc}
0 & 0 & t^{-1} \\
0 & t^{-2}(1+\lambda \rho)^{-2} g^{i j} & 0 \\
t^{-1} & 0 & -2 t^{-2} \rho
\end{array}\right)
$$

and the Christoffel symbol of $\tilde{g}$ is given by

$$
\begin{gathered}
\tilde{\Gamma}_{I J}^{0}=\left(\begin{array}{ccc}
0 & 0 & 0 \\
0 & -\lambda t(1+\lambda \rho) g_{i j} & 0 \\
0 & 0 & 0
\end{array}\right), \quad \tilde{\Gamma}_{I J}^{k}=\left(\begin{array}{ccc}
0 & t^{-1} \delta_{j}{ }^{k} & 0 \\
t^{-1} \delta_{i}{ }^{k} & \Gamma^{k}{ }_{i j} & \lambda(1+\lambda \rho)^{-1} \delta_{i}{ }^{k} \\
0 & \lambda(1+\lambda \rho)^{-1} \delta_{j}{ }^{k} & 0
\end{array}\right), \\
\tilde{\Gamma}_{I J}^{\infty}=\left(\begin{array}{ccc}
0 & -(1+\lambda \rho)(1-\lambda \rho) g_{i j} & 0 \\
0 & 0 & 0
\end{array}\right) .
\end{gathered}
$$

A direct computation shows that $\tilde{W}_{i j k l}=t^{2} W_{i j k l}$, where $\tilde{W}$ and $W$ are the Weyl tensors of $\tilde{g}$ and $g$, respectively (the latter is extended to $\tilde{G}=\mathbb{R}_{+} \times M \times \mathbb{R}$ in the trivial way). The other components of $\tilde{W}$ are zero.

Lemma 4.1. Let $\tilde{g}$ be as above, and suppose that $\tilde{\sigma} \in \tilde{\mathscr{T}}(w)$ is of the form

$$
\tilde{\sigma}_{i j}=t^{w}(1+\lambda \rho)^{w} \sigma_{i j},
$$

where $\sigma_{i j}$ is a symmetric 2-tensor on $(M, g)$. Then

$$
\tilde{\Delta}_{\mathrm{L}} \tilde{\sigma}=t^{w-2}(1+\lambda \rho)^{w-2}\left(\Delta_{\mathrm{L}}-4(n-1) \lambda-2(w-2)(n+w-3) \lambda\right) \sigma,
$$

where $\Delta_{\mathrm{L}}=\Delta+4 n \lambda-2 \mathscr{W}^{\circ}$ is the Lichnerowicz Laplacian of $g$. 
Proof. The first covariant derivative of $\tilde{\sigma}$ is as follows:

$$
\begin{aligned}
\tilde{\nabla}_{\infty} \tilde{\sigma}_{i j} & =\partial_{\rho} \tilde{\sigma}_{i j}-2 \tilde{\Gamma}^{k}{ }_{\infty(i} \tilde{\sigma}_{j) k}=t^{w}(1+\lambda \rho)^{w-1}(w-2) \lambda \sigma_{i j}, \\
\tilde{\nabla}_{0} \tilde{\sigma}_{i j} & =\partial_{t} \tilde{\sigma}_{i j}-2 \tilde{\Gamma}^{k}{ }_{0(i} \tilde{\sigma}_{j) k}=t^{w-1}(1+\lambda \rho)^{w}(w-2) \sigma_{i j}, \\
\tilde{\nabla}_{k} \tilde{\sigma}_{i j} & =\partial_{x^{k}} \tilde{\sigma}_{i j}-2 \tilde{\Gamma}_{k(i}^{l} \tilde{\sigma}_{j) l}=t^{w}(1+\lambda \rho)^{w} \nabla_{k} \sigma_{i j}, \\
\tilde{\nabla}_{k} \tilde{\sigma}_{i \infty} & =-\tilde{\Gamma}_{k \infty}^{l} \tilde{\sigma}_{i l}=-t^{w}(1+\lambda \rho)^{w-1} \lambda \sigma_{i k}, \\
\tilde{\nabla}_{k} \tilde{\sigma}_{i 0} & =-\tilde{\Gamma}_{k 0}^{l} \tilde{\sigma}_{i l}=-t^{w-1}(1+\lambda \rho)^{w} \sigma_{i k} .
\end{aligned}
$$

Therefore,

$$
\begin{aligned}
\tilde{\nabla}_{0} \tilde{\nabla}_{\infty} \tilde{\sigma}_{i j} & =\partial_{t} \tilde{\nabla}_{\infty} \tilde{\sigma}_{i j}-\tilde{\Gamma}_{0 \infty}^{\infty} \tilde{\nabla}_{\infty} \tilde{\sigma}_{i j}-2 \tilde{\Gamma}_{0(i \mid}^{k} \tilde{\nabla}_{\infty} \tilde{\sigma}_{\mid j) k} \\
& =t^{w-1}(1+\lambda \rho)^{w-1}(w-2)(w-3) \lambda \sigma_{i j}, \\
\tilde{\nabla}_{\infty} \tilde{\nabla}_{0} \tilde{\sigma}_{i j}= & \tilde{\nabla}_{0} \tilde{\nabla}_{\infty} \tilde{\sigma}_{i j}-2 \tilde{R}_{\infty 0}{ }_{(i} \tilde{\sigma}_{j) k}=\tilde{\nabla}_{0} \tilde{\nabla}_{\infty} \tilde{\sigma}_{i j}, \\
\tilde{\nabla}_{\infty} \tilde{\nabla}_{\infty} \tilde{\sigma}_{i j}= & \partial_{\rho} \tilde{\nabla}_{\infty} \tilde{\sigma}_{i j}-2 \tilde{\Gamma}_{\infty(i \mid}^{k} \tilde{\nabla}_{\infty} \tilde{\sigma}_{\mid j) k}=t^{w}(1+\lambda \rho)^{w-2}(w-2)(w-3) \lambda^{2} \sigma_{i j}, \\
g^{k l} \tilde{\nabla}_{k} \tilde{\nabla}_{l} \tilde{\sigma}_{i j}= & \partial_{x^{k}} \tilde{\nabla}_{l} \tilde{\sigma}_{i j}-\tilde{\Gamma}_{k l}^{m} \tilde{\nabla}_{m} \tilde{\sigma}_{i j}-2 \tilde{\Gamma}_{k(i \mid}^{m} \tilde{\nabla}_{l} \tilde{\sigma}_{\mid j) m} \\
& \quad-\tilde{\Gamma}_{k l}^{\infty} \tilde{\nabla}_{\infty} \tilde{\sigma}_{i j}-2 \tilde{\Gamma}_{k(i \mid}^{\infty} \tilde{\nabla}_{l} \tilde{\sigma}_{\mid j) \infty}-\tilde{\Gamma}_{k l}^{0} \tilde{\nabla}_{0} \tilde{\sigma}_{i j}-2 \tilde{\Gamma}_{k(i \mid}^{0} \tilde{\nabla}_{l} \tilde{\sigma}_{\mid j) 0} \\
= & -t^{w}(1+\lambda \rho)^{w}\left(\Delta \sigma_{i j}-2(n(w-2)-2) \lambda \sigma_{i j}\right)
\end{aligned}
$$

and hence

$$
\begin{aligned}
\tilde{\Delta} \tilde{\sigma}_{i j} & =-2 t^{-1} \tilde{\nabla}_{0} \tilde{\nabla}_{\infty} \tilde{\sigma}_{i j}+2 t^{-2} \rho \tilde{\nabla}_{\infty} \tilde{\nabla}_{\infty} \tilde{\sigma}_{i j}-t^{-2}(1+\lambda \rho)^{-2} g^{k l} \tilde{\nabla}_{k} \tilde{\nabla}_{l} \tilde{\sigma}_{i j} \\
& =t^{w-2}(1+\lambda \rho)^{w-2}(\Delta+4 \lambda-2(w-2)(n+w-3) \lambda) \sigma_{i j} .
\end{aligned}
$$

Consequently, $\tilde{\Delta}_{\mathrm{L}} \tilde{\sigma}=(\tilde{\Delta}-2 \stackrel{\mathscr{W}}{)} \tilde{\sigma}$ is given by (4-2).

Theorem 4.2. Let $(M,[g])$ be a conformally Einstein manifold with $\operatorname{dim} M=n \geq 3$, and $g \in[g]$ an Einstein representative with Schouten tensor $P_{i j}=\lambda g_{i j}$. Then, the action of $P_{k}$ restricted to $\mathscr{Y}_{\mathrm{TT}}^{g}(-n / 2+2+k)$ is given by $(0-5)$.

Proof. Let $\varphi=t^{-n / 2+2+k} \bar{\varphi} \in \mathscr{G}_{\mathrm{TT}}^{g}(-n / 2+2+k)$ and $\tilde{\sigma}=(1+\lambda \rho)^{-n / 2+2+k} \varphi$. Then

$$
\tilde{\nabla}_{k} \tilde{\sigma}_{i j}=t^{-n / 2+2+k}(1+\lambda \rho)^{-n / 2+2+k} \nabla_{k} \bar{\varphi}_{i j}, \quad \tilde{\nabla}_{\infty} \tilde{\sigma}_{0 i}=\tilde{\nabla}_{0} \tilde{\sigma}_{\infty i}=\tilde{\nabla}_{\infty} \tilde{\sigma}_{\infty i}=0 .
$$

Since $\bar{\varphi}$ is a TT-tensor on $(M, g), \tilde{\sigma}$ itself is a TT-tensor with respect to $\tilde{g}$, and hence is an extension of the ambient lift of $\varphi$. We may compute $\tilde{\Delta}_{\mathrm{L}}^{k} \tilde{\sigma}$ by Lemma 4.1. By taking the value along $\mathscr{G}$ and trivializing with respect to $g$, we obtain (0-5).

Now we prove our main theorem.

Proof of Theorem 0.2. Let $\varphi=\mathscr{K}_{[g]} \xi+\varphi_{\mathrm{TT}}^{g}$ be the decomposition of $\varphi=\left.\dot{\boldsymbol{g}}_{t}\right|_{t=0}$ with respect to (0-1) and $\Xi_{t}$ the flow generated by $\xi$. Then $\boldsymbol{g}_{t}^{\prime}=\Xi_{-t}^{*} \boldsymbol{g}_{t}$ satisfies 
$\left.\dot{\boldsymbol{g}}_{t}^{\prime}\right|_{t=0}=\varphi_{\mathrm{TT}}^{g}$ and the total $Q$-curvature of $\boldsymbol{g}_{t}^{\prime}$ is equal to $\bar{Q}_{t}$. Therefore

$$
\left.\frac{d^{2}}{d t^{2}} \bar{Q}_{t}\right|_{t=0}=\left.\frac{d^{2}}{d t^{2}} \bar{Q}_{t}^{\prime}\right|_{t=0}=-\frac{1}{4} \int_{M}\left\langle P \varphi_{\mathrm{TT}}^{g}, \varphi_{\mathrm{TT}}^{g}\right\rangle,
$$

and thus (0-2) follows from Theorem 4.2. Under the assumption of the latter half of the theorem, any eigenvalue of $\left.\Delta_{\mathrm{L}}\right|_{\mathscr{S}_{\mathrm{TT}(2)}^{g}}-4(n-1) \lambda+4 m(n-2 m-1) \lambda$ is strictly positive for $0 \leq m \leq n / 2-1$. Therefore, if $\varphi_{\mathrm{TT}}^{g} \neq 0$, the second derivative of $\bar{Q}_{t}$ at $t=0$ is negative.

\section{Acknowledgements}

I wish to thank Kengo Hirachi for the suggestion to take a variational approach to the $Q$-curvature and for insightful advice, and Robin Graham for discussions on our formula (0-2) and for letting me know about the work [Møller and Ørsted 2009], which is based on [Branson et al. 1996]; see also the abstract by R. Graham in [Oberwolfach 2005, 2200-2203]. I also thank Bent Ørsted for related discussions. Moreover, Colin Guillarmou informed me that he has given another proof of (0-2) with Sergiu Moroianu and Jean-Marc Schlenker in [Guillarmou et al. 2012].

\section{References}

[Besse 1987] A. L. Besse, Einstein manifolds, Ergebnisse der Math. und ihrer Grenzgebiete (3) 10, Springer, Berlin, 1987. MR 88f:53087 Zbl 0613.53001

[Branson 1995] T. P. Branson, "Sharp inequalities, the functional determinant, and the complementary series”, Trans. Amer. Math. Soc. 347:10 (1995), 3671-3742. MR 96e:58162 Zbl 0848.58047

[Branson 2005] T. Branson, " $Q$-curvature and spectral invariants", Rend. Circ. Mat. Palermo (2) Suppl. 75 (2005), 11-55. MR 2006h:53024 Zbl 1101.53016

[Branson and Gover 2005] T. Branson and A. R. Gover, "Conformally invariant operators, differential forms, cohomology and a generalisation of $Q$-curvature", Comm. Partial Differential Equations 30:10-12 (2005), 1611-1669. MR 2006k:58060 Zbl 1226.58011

[Branson and Gover 2007] T. P. Branson and A. R. Gover, "The conformal deformation detour complex for the obstruction tensor", Proc. Amer. Math. Soc. 135:9 (2007), 2961-2965. MR 2008j:58029 Zbl 1126.53023

[Branson and Gover 2008] T. P. Branson and A. R. Gover, "Origins, applications and generalisations of the $Q$-curvature”, Acta Appl. Math. 102:2-3 (2008), 131-146. MR 2010a:53025 Zbl 1145.53022

[Branson et al. 1996] T. Branson, G. Ólafsson, and B. Ørsted, "Spectrum generating operators and intertwining operators for representations induced from a maximal parabolic subgroup", J. Funct. Anal. 135:1 (1996), 163-205. MR 97g:22009 Zbl 0841.22011

[Fefferman and Graham 1985] C. Fefferman and C. R. Graham, "Conformal invariants", pp. 95-116 in The mathematical heritage of Élie Cartan (Lyon, 1984), Astérisque (numéro hors série), Société Mathématique de France, Paris, 1985. MR 87g:53060 Zbl 0602.53007

[Fefferman and Graham 2012] C. Fefferman and C. R. Graham, The ambient metric, Annals of Mathematics Studies 178, Princeton University Press, 2012. MR 2858236 Zbl 1243.53004 
[Gover and Peterson 2006] A. R. Gover and L. J. Peterson, "The ambient obstruction tensor and the conformal deformation complex”, Pacific J. Math. 226:2 (2006), 309-351. MR 2007m:58029 Zbl 1125.53010

[Graham and Hirachi 2005] C. R. Graham and K. Hirachi, "The ambient obstruction tensor and $Q$-curvature", pp. 59-71 in AdS/CFT correspondence: Einstein metrics and their conformal boundaries, edited by O. Biquard, IRMA Lect. Math. Theor. Phys. 8, Eur. Math. Soc., Zürich, 2005. MR 2006k:53045 Zbl 1074.53027

[Graham et al. 1992] C. R. Graham, R. Jenne, L. J. Mason, and G. A. J. Sparling, "Conformally invariant powers of the Laplacian, I: Existence", J. London Math. Soc. (2) 46:3 (1992), 557-565. MR 94c:58226 Zbl 0726.53010

[Guillarmou et al. 2012] C. Guillarmou, S. Moroianu, and J.-M. Schlenker, "The renormalized volume and uniformisation of conformal structures", preprint, 2012. arXiv 1211.6705

[Møller and Ørsted 2009] N. M. Møller and B. Ørsted, "Rigidity of conformal functionals on spheres", preprint, 2009. arXiv 0902.4067

[Oberwolfach 2005] J. Brüning, R. Mazzeo and P. Piazza (organizers), "Analysis and geometric singularities: Abstracts from the workshop held August 21-27, 2005", Oberwolfach Rep. 2:3 (2005), 2187-2239. MR 2079248

Received February 15, 2012. Revised January 10, 2013.

YoshiHIKO MATSUMOTO

GRADUATE SCHOOL OF MATHEMATICAL SCIENCES

THE UNIVERSITY OF TOKYO

3-8-1 Komaba, Meguro-Ku

TOKYO 153-8914

JAPAN

yoshim@ms.u-tokyo.ac.jp 


\title{
PACIFIC JOURNAL OF MATHEMATICS
}

\author{
msp.org/pjm
}

Founded in 1951 by E. F. Beckenbach (1906-1982) and F. Wolf (1904-1989)

\section{EDITORS}

V. S. Varadarajan (Managing Editor)

Department of Mathematics

University of California

Los Angeles, CA 90095-1555

pacific@math.ucla.edu

Paul Balmer

Department of Mathematics

University of California

Los Angeles, CA 90095-1555

balmer@math.ucla.edu

Daryl Cooper

Department of Mathematics

University of California

Santa Barbara, CA 93106-3080 cooper@math.ucsb.edu

Jiang-Hua $\mathrm{Lu}$

Department of Mathematics

The University of Hong Kong

Pokfulam Rd., Hong Kong jhlu@maths.hku.hk
Don Blasius

Department of Mathematics University of California

Los Angeles, CA 90095-1555

blasius@math.ucla.edu

Robert Finn

Department of Mathematics Stanford University

Stanford, CA 94305-2125

finn@math.stanford.edu

Sorin Popa

Department of Mathematics

University of California

Los Angeles, CA 90095-1555

popa@math.ucla.edu

Paul Yang

Department of Mathematics

Princeton University

Princeton NJ 08544-1000

yang@math.princeton.edu

\section{PRODUCTION}

Silvio Levy, Scientific Editor, production@msp.org

\section{SUPPORTING INSTITUTIONS}

ACADEMIA SINICA, TAIPEI

CALIFORNIA INST. OF TECHNOLOGY

INST. DE MATEMÁTICA PURA E APLICADA

KEIO UNIVERSITY

MATH. SCIENCES RESEARCH INSTITUTE

NEW MEXICO STATE UNIV.

OREGON STATE UNIV.

\author{
STANFORD UNIVERSITY \\ UNIV. OF BRITISH COLUMBIA \\ UNIV. OF CALIFORNIA, BERKELEY \\ UNIV. OF CALIFORNIA, DAVIS \\ UNIV. OF CALIFORNIA, LOS ANGELES \\ UNIV. OF CALIFORNIA, RIVERSIDE \\ UNIV. OF CALIFORNIA, SAN DIEGO \\ UNIV. OF CALIF., SANTA BARBARA
}

\author{
Vyjayanthi Chari \\ Department of Mathematics \\ University of California \\ Riverside, CA 92521-0135 \\ chari@math.ucr.edu \\ Kefeng Liu \\ Department of Mathematics \\ University of California \\ Los Angeles, CA 90095-1555 \\ liu@math.ucla.edu \\ Jie Qing \\ Department of Mathematics \\ University of California \\ Santa Cruz, CA 95064 \\ qing@cats.ucsc.edu
}

These supporting institutions contribute to the cost of publication of this Journal, but they are not owners or publishers and have no responsibility for its contents or policies.

See inside back cover or msp.org/pjm for submission instructions.

The subscription price for 2013 is US \$400/year for the electronic version, and \$485/year for print and electronic.

Subscriptions, requests for back issues and changes of subscribers address should be sent to Pacific Journal of Mathematics, P.O. Box 4163, Berkeley, CA 94704-0163, U.S.A. The Pacific Journal of Mathematics is indexed by Mathematical Reviews, Zentralblatt MATH, PASCAL CNRS Index, Referativnyi Zhurnal, Current Mathematical Publications and the Science Citation Index.

The Pacific Journal of Mathematics (ISSN 0030-8730) at the University of California, c/o Department of Mathematics, 798 Evans Hall \#3840, Berkeley, CA 94720-3840, is published monthly except July and August. Periodical rate postage paid at Berkeley, CA 94704, and additional mailing offices. POSTMASTER: send address changes to Pacific Journal of Mathematics, P.O. Box 4163, Berkeley, CA 94704-0163.

PJM peer review and production are managed by EditFLOW ${ }^{\circledR}$ from Mathematical Sciences Publishers.

PUBLISHED BY

mathematical sciences publishers

nonprofit scientific publishing

http://msp.org/

(C) 2013 Mathematical Sciences Publishers 


\section{PACIFIC JOURNAL OF MATHEMATICS}

Volume $262 \quad$ No. $2 \quad$ April 2013

Certifying incompressibility of noninjective surfaces with scl

DANNY CALEGARI

Global well-posedness for the 3D rotating Navier-Stokes equations with highly oscillating initial data

QIONGLEI CHEN, CHANGXING MiAO and ZHIFEI ZHANG

Presenting Schur superalgebras

HOUSSEIN El TURKEY and JONATHAN R. KUJAWA

Classifying zeros of two-sided quaternionic polynomials and computing zeros of two-sided polynomials with complex coefficients

FENG LIANGGUI and ZHAO KAIMING

Coxeter groups, imaginary cones and dominance

XIANG FU

Semicontinuity of automorphism groups of strongly pseudoconvex domains: The low differentiability case

Robert E. Greene, Kang-Tae Kim, Steven G. Krantz and

AERYEONG SEO

Klein four-subgroups of Lie algebra automorphisms

JING-SONG HUANG and JUN YU

Fractal entropy of nonautonomous systems

Rui KuANG, WEN-ChiaO CHENG and BING LI

A GJMS construction for 2-tensors and the second variation of the total

$Q$-curvature

YoshiniKo MaTSUMOTO

Droplet condensation and isoperimetric towers

Matteo Novaga, Andrei Sobolevski and Eugene Stepanov

Brauer's height zero conjecture for metacyclic defect groups

BENJAMIN SAMBALE 